\title{
Impact of Cold-Water Immersion Compared with Passive Recovery Following a Single Bout of Strenuous Exercise on Athletic Performance in Physically Active Participants: A Systematic Review with Meta-analysis and Meta-regression
}

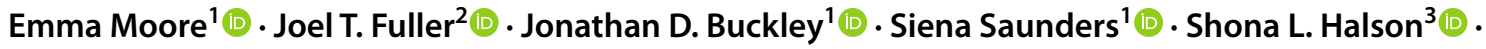 \\ James R. Broatch ${ }^{4}$ (i) . Clint R. Bellenger ${ }^{1}[$ (D)
}

Accepted: 9 January 2022 / Published online: 14 February 2022

(c) The Author(s) 2022

\begin{abstract}
Background Studies investigating the effects of cold-water immersion (CWI) on the recovery of athletic performance, perceptual measures and creatine kinase (CK) have reported mixed results in physically active populations.

Objectives The purpose of this systematic review was to investigate the effects of CWI on recovery of athletic performance, perceptual measures and CK following an acute bout of exercise in physically active populations.

Study Design Systematic review with meta-analysis and meta-regression.

Methods A systematic search was conducted in September 2021 using Medline, SPORTDiscus, Scopus, Web of Science, Cochrane Library, EmCare and Embase databases. Studies were included if they were peer reviewed and published in English, included participants who were involved in sport or deemed physically active, compared CWI with passive recovery methods following an acute bout of strenuous exercise and included athletic performance, athlete perception and CK outcome measures. Studies were divided into two strenuous exercise subgroups: eccentric exercise and high-intensity exercise. Random effects meta-analyses were used to determine standardised mean differences (SMD) with 95\% confidence intervals. Meta-regression analyses were completed with water temperature and exposure durations as continuous moderator variables. Results Fifty-two studies were included in the meta-analyses. CWI improved the recovery of muscular power $24 \mathrm{~h}$ after eccentric exercise (SMD 0.34 [95\% CI 0.06-0.62]) and after high-intensity exercise (SMD 0.22 [95\% CI 0.004-0.43]), and reduced serum CK (SMD - 0.85 [95\% CI - 1.61 to - 0.08]) $24 \mathrm{~h}$ after high-intensity exercise. CWI also improved muscle soreness (SMD - 0.89 [95\% CI - 1.48 to - 0.29]) and perceived feelings of recovery (SMD 0.66 [95\% CI 0.29-1.03]) 24 h after high-intensity exercise. There was no significant influence on the recovery of strength performance following either eccentric or high-intensity exercise. Meta-regression indicated that shorter time and lower temperatures were related to the largest beneficial effects on serum CK (duration and temperature dose effects) and endurance performance (duration dose effects only) after high-intensity exercise.

Conclusion CWI was an effective recovery tool after high-intensity exercise, with positive outcomes occurring for muscular power, muscle soreness, CK, and perceived recovery $24 \mathrm{~h}$ after exercise. However, after eccentric exercise, CWI was only effective for positively influencing muscular power $24 \mathrm{~h}$ after exercise. Dose-response relationships emerged for positively influencing endurance performance and reducing serum CK, indicating that shorter durations and lower temperatures may improve the efficacy of CWI if used after high-intensity exercise.

Funding Emma Moore is supported by a Research Training Program (Domestic) Scholarship from the Australian Commonwealth Department of Education and Training.
\end{abstract}

Protocol registration Open Science Framework: 10.17605/OSF.IO/SRB9D.

Extended author information available on the last page of the article 


\section{Key Points}

Cold-water immersion is more likely to positively influence muscular power performance, but not muscular strength performance.

Cold-water immersion is more likely to positively influence muscular power performance, muscle soreness, serum creatine kinase, and perceived recovery after highintensity exercise when compared with passive recovery.

Dose-response relationships indicate that lower temperature cold-water immersion may be more effective after high-intensity exercise for removal of serum creatine kinase.

Dose-response relationships indicate shorter duration cold-water immersion may be more effective after high-intensity exercise for endurance performance and removal of serum creatine kinase.

\section{Introduction}

During acute (short-term) phases of training and competition, athletes are required to maximise their performance in competition while managing fatigue and soreness [1]. Therefore, recovery between exercise bouts is paramount to prepare for subsequent training sessions or competition events [2] and failure to adequately recover may lead to fatigue and reductions in performance [3]. Therefore, techniques have been developed to accelerate recovery and optimise performance, especially in scenarios where time for recovery may be limited, with the goal of assisting athletes to reach their peak performance in competition. Cold-water immersion (CWI) is a commonly performed recovery technique that can be cost effective and able to be utilised in many environments [4]. Studies have found that CWI is one of the most effective recovery strategies implemented by athletes, with an effectiveness rating of 4.3/5 [5].

The beneficial effects of CWI in recovery are thought to be largely mediated by increased hydrostatic pressure and/or a reduction in body/tissue temperature [6]. Resultant alterations in tissue blood flow, post-exercise fluid retention and metabolic activity may accelerate the recovery process via the reduction of muscle damage, swelling and inflammation, muscle spasm, pain, and thermal strain [4]. CWI may also improve mood due to endorphin release [7] and enhance perceptions of recovery by reducing muscular soreness via an analgesic effect [1].

Recently completed meta-analyses investigating the efficacy of CWI for promoting recovery from an acute bout of exercise have reported performance, perceptual and physiological benefits of CWI compared with passive recovery [8-11]. However, one review was limited in that it combined multiple timepoints of recovery (i.e. $24 \mathrm{~h}$, $48 \mathrm{~h}, 72 \mathrm{~h}$ etc.) within a single analysis [10] such that the investigators could not identify a time course of recovery. These reviews also pooled data from multiple cooling methods (i.e. CWI, cooling packs, cryotherapy chambers) [10], and different exercise intervention modes (i.e. eccentric exercise and high-intensity exercise) [9-11], and both crossover and parallel studies with no consideration of the statistical differences between study designs. For example, many crossover studies do not report within-participant differences and provide only mean values for each treatment group, which reduces the precision of the results [12]. These variations resulted in high levels of heterogeneity within the analyses, and only one review attempted sub-group analyses to reduce heterogeneity, with limited effect [8]. While the time-based ( $24 \mathrm{~h}, 48 \mathrm{~h}, 72 \mathrm{~h})$ and exercise intervention-based (eccentric and high-intensity exercise) subgroups showed some positive effects of CWI, in most of the analyses the heterogeneity was not reduced, and the low study numbers $(<3)$ in some subgroups minimised conclusions that could be made.

One review [9] attempted to elucidate whether there was a dose-response relationship between muscle soreness and CWI temperature or exposure duration in trained and untrained participants. However, that review arbitrarily clustered temperatures and durations into small sub-metaanalyses. This sub-meta-analyses approach combines results to obtain a summarised mean difference that only takes into account the methodological differences between studies (heterogeneity) $[13,14]$. This review identified an 'optimum' CWI protocol of $11-15{ }^{\circ} \mathrm{C}$ for $11-15$ min based on small study numbers ( $<4$ per sub-analysis), and this protocol has been subsequently used as the guideline for recovery prescription across various sporting populations despite the limited evidence. This review also only focused on the reduction of muscle soreness, ignoring other parameters of recovery. When making recommendations for appropriate protocols, the strength of evidence should incorporate more than just heterogeneity, but also include the number of participants and level of bias within the studies such as is seen in the Grading of Recommendations Assessment, Development and Evaluation (GRADE) method of grading evidence quality and strength of recommendations [15]. Therefore, the small study numbers (and subsequently participant numbers) weaken any definitive conclusions.

The aim of this review was to investigate the impact of CWI on recovery of physiological, perceptual, and athletic performance variables following an acute bout of strenuous exercise in physically active participants. Additionally, this review aimed to describe the recovery of these variables across multiple timepoints following exercise of differing 
modality, as well as identify dose-response effects of temperature and exposure duration using meta-regression. This review also aimed to provide systematic recommendations using GRADE criteria. Identifying protocols that aid recovery of specific athletic performance outcomes following strenuous exercise will allow appropriate prescription of CWI protocols for physically active individuals who wish to accelerate their recovery following exercise.

\section{Methods}

\subsection{Design}

This review followed the Preferred Reporting Items for Systematic Reviews and Meta-Analysis (PRISMA) statement for the reporting of systematic reviews and meta-analyses [16] and was prospectively registered with Open Science Framework (https://doi.org/10.17605/OSF.IO/SRB9D).

\subsection{Search Strategy and Selection Criteria}

Medline, SPORTDiscus, Scopus, Web of Science, Cochrane Library, EmCare and Embase databases were searched from inception until 20 September 2021 using the following search strategy, which was adapted for each database:

athlet* or sport* or exerci* or football* or soccer or hockey or basketball* or netball* or volleyball* or "track and field" or cycli* or running or runner* or swim* or handball or softball* or tennis or baseball or cross country or cricket or surf* or skiing or golf or hurdling or bicycling or boxing or gymnast* or martial arts or racquet sports or badminton or jogg* or walk* or weight lifting or lift* weights or weight?lift* or wrestling or resistance train* or endurance train* or interval train* or climb* or strength* train* or strength* program and (cold* or ice* or low* temp*) adj3 (bath* or hydrotherap* or immers* or submers* or submerg*)

Individual sport terms were included in the search strategy to ensure that studies that used sport simulations, training or games were captured in the search process; this ensured a vigorous search was completed.

Database search results were exported to Endnote ${ }^{\circledR}$ (version 9.2, Thomson-Reuters, Toronto, CA, USA) and then uploaded to Covidence ${ }^{\odot}$ Systematic Review software (Veritas Health Innovations, Melbourne, VIC, Australia). All duplicates were removed before two reviewers independently screened titles and abstracts for eligibility. Full texts were obtained for the remaining articles and independently assessed for eligibility by two reviewers (EM, SS). Results from each reviewer were compared after each stage and any discrepancies were resolved by an independent reviewer. Reference lists of all eligible studies and any previous systematic reviews were checked to identify additional eligible studies that were not identified by the primary search (i.e. pearling).

Inclusion criteria were as follows: (i) peer-reviewed, randomised controlled trials published in the English language, (ii) participants were aged over 18 years and performed exercise regularly, (iii) protocols that used CWI within $15 \mathrm{~min}$ following a single bout of strenuous exercise (defined by the authors as an exercise bout that would cause muscle damage) with further immersions permitted to be completed on subsequent days, (iv) used passive recovery (no recovery intervention) as the comparator intervention (to determine the influence of CWI on recovery measures), (v) outcome measures included time to recovery of exercise performance (endurance, flexibility, muscular strength, muscular power [including jump performance, anaerobic power performance of $<10 \mathrm{~s}$ or sprint performance]) or physiological (creatine kinase $[\mathrm{CK}]$ ) and perceptual markers of recovery (delayed onset muscle soreness [DOMS], perceived recovery) and (vi) outcome measures were performed at the following timepoints: $1 \mathrm{~h}$ after exercise, $24 \mathrm{~h}$ after exercise, $48 \mathrm{~h}$ after exercise, $72 \mathrm{~h}$ after exercise, $96 \mathrm{~h}$ after exercise and $168 \mathrm{~h}$ after exercise. Studies were excluded if they used combined treatments that may confound CWI results (e.g. combining CWI with compression garments, CWI with active recovery, CWI with nutritional supplements), training interventions involving more than one session of exercise, there were insufficient data to perform analysis or if data were presented in formats such as theses or conference abstracts.

\subsection{Risk of Bias}

An assessment of methodological quality for the selected studies was undertaken using the randomised controlled trial (RCT) checklist from the Scottish Intercollegiate Guidelines Network (SIGN) [17]. The SIGN RCT checklist was developed to ensure a balance between methodological quality and practicality of use for authors and was used in the present review because it is specific to the design of included studies. Before commencing assessment, definitions provided by SIGN were clarified by the review team. Two reviewers (EM, SS) appraised each study based on these appraisal definitions, with any discrepancies resolved by an independent reviewer (JB). A grade of 'yes', 'no', 'can't say' or 'not applicable' was issued for each appraisal item. 'Yes' and 'not applicable' answers were indicative of a lower risk of bias; therefore, the total frequency of 'yes' and 'not applicable' answers were tallied to indicate overall methodological quality. The quality of each study was labelled as 'high quality', 'acceptable', 'low quality' or 'unacceptable'. 


\subsection{Data Extraction}

Data were extracted by one reviewer (EM) and entered in a standardised Microsoft Excel ${ }^{\odot}$ spreadsheet (V2105, Microsoft, Washington, USA). These data were independently crosschecked by another reviewer (SS) and any discrepancies were resolved through discussion. Further information was sought from study authors if all information could not be obtained from the full-text article. The extracted information included publication details (author information, publication date, country of origin), study methodology (sample size, exercise intervention, study type, assessment measures, comparison intervention), participant information (age, sex, height, body mass, sport, training history), CWI protocol (temperature, duration, number of immersions, depth of immersion, body position during immersion, timing of immersion post-exercise), passive recovery protocol (temperature of environment, duration, body position during protocol), and assessment measures (test, units, measurements at various timepoints, effect sizes, confidence intervals, $p$-values).

\subsection{Statistical Considerations}

Summary effect sizes were presented for each study using standardised mean difference (SMD) for comparing the effect between CWI and passive recovery. SMDs were calculated using Hedges' h correction for positive bias. The precision of the effect sizes was described using $95 \%$ confidence intervals (CI) whenever sufficient information was provided by the study authors. For the purpose of this review, effect sizes were presented for each study and were considered trivial (SMD < 0.2), small (SMD 0.20-0.60), moderate (SMD 0.61-1.20), large (SMD 1.21-2.00) and very large $(\mathrm{SMD}>2.01)$ [18].

Random-effects meta-analyses and meta-regression were performed using restricted maximum likelihood estimation with the Metafor statistical package in $\mathrm{R}$ software (version 3.4.3, R Foundation for Statistical Computing). Weighting of study effects was based on the inverse variance method. Separate analyses were performed for exercise mode subgroups (high-intensity exercise vs resistance-based eccentric muscle contraction) to identify whether the effects of differing exercise modalities alter the recovery timeline. Both CWI duration and temperature were considered as continuous moderator variables due to the various combinations of duration and temperature evident in the data synthesis. Study ID was included as a random factor to account for studies that reported multiple CWI versus passive recovery comparisons (i.e. different CWI temperatures).

The methods described by Elbourne et al. [12] were used to combine data from parallel and crossover studies in the meta-analyses whenever possible. This was possible for crossover studies that reported treatment effect with an accompanying confidence interval, standard error, or $p$-value from an appropriate statistical analysis of paired data. Crossover studies that did not provide this information (i.e. they only reported mean and standard deviation for each treatment condition) were also included if it was possible to estimate the standard error of the paired difference using information available from other included crossover studies of the same outcome measure. This estimation process is described in detailed by Elbourne et al. [12]. In brief, the correlation between treatment and comparison conditions can be estimated from the included crossover studies that provide complete information from paired analysis. This estimated correlation value can then be used to impute a standard error for the treatment effect from crossover studies that do not provide information about treatment effect variance. The lowest available correlation was used for this imputation when multiple correlations were available for a review outcome.

Muscular power outcomes for jump, sprint and anaerobic power of $<10 \mathrm{~s}$ were pooled for the purpose of analysis to increase study numbers. Despite potential differences in the kinetics of recovery, it was considered appropriate to combine these outcomes to evaluate the effects on recovery of the phosphagen energy system.

Statistical heterogeneity within each meta-analysis was investigated using $I^{2}$ statistics, which indicated the consistency of the study effects between the included studies [19]. Statistical heterogeneity (i.e. inconsistency) was considered low $\left(I^{2}<25 \%\right)$, moderate $\left(I^{2} 25-49 \%\right)$ and high $\left(I^{2}>50 \%\right)$ [19]. The overall quality of the evidence synthesis was rated high, moderate, low or very low using the GRADE system [15]. The quality rating was downgraded one level from high for each of the following limitations: total number of unique participants was < 100 (imprecision), high statistical heterogeneity (inconsistency) and more than $50 \%$ of the studies in the meta-analysis deemed to be low quality.

\section{Results}

\subsection{Search Results}

Systematic database searches identified 4849 potential studies and pearling of previous reviews identified six more potential studies for inclusion. Following the removal of duplicates and ineligible articles, 52 studies were included in this review. Several studies met the inclusion criteria but were excluded due to missing/unusable data (where authors were not able to be contacted) or outcomes measured at timepoints not included in the meta-analysis [20-38]. A complete overview of the screening process can be found in 
Fig. 1. A complete overview of articles identified for inclusion in the review can be found in Table 1 .

\subsection{Risk of Bias}

Based upon the agreed criteria of the SIGN RCT checklist, reviewers deemed 11 (21\%) studies to be high quality [42, $50,51,54,57,58,60,66,77,79,89], 37$ (69\%) to be acceptable quality [39-41, 43-49, 52, 53, 55, 56, 59, 61, 64, 65, $67-76,78,80-84,86-88]$ and four (10\%) studies to be low quality $[62,63,85,90]$. The noteworthy results from the risk of bias analysis indicated that concealment of the treatment groups from the research group was rarely completed; only four studies concealed treatment groups from the research team [50, 57, 66, 89]. Reporting of randomisation protocols of treatment groups was also poor for most studies; only 11 studies adequately reported randomisation protocols [42, $50,51,54,57,58,60,66,77,79,89]$. Results of the risk of bias analysis for each individual study can be found in
Online Supplement 1 of the electronic supplementary material (ESM). No study reported any conflict of interest.

\subsection{Meta-analysis}

\subsubsection{The Effects of Cold-Water Immersion (CWI) on Recovery of Power Performance}

CWI was effective in promoting the recovery of muscular power $24 \mathrm{~h}$ after eccentric exercise (Fig. 2; Table 2; small effects; $p=0.018$; GRADE $=$ high). There were significant small effects at $48 \mathrm{~h}$, moderate effects at $72 \mathrm{~h}$ (Table 2; both timepoints GRADE = high) and non-significant small effects at 96 and $168 \mathrm{~h}$ (Table 2; both timepoints GRADE = moderate) in favour of CWI. Water temperature and exposure duration had no significant moderating effects at any timepoint.

CWI was effective in promoting the recovery of muscular power $24 \mathrm{~h}$ after high-intensity exercise (Fig. 2; Table 2; small effects; $p=0.046$; GRADE $=$ moderate). Other timepoints found non-significant small effects in favour of

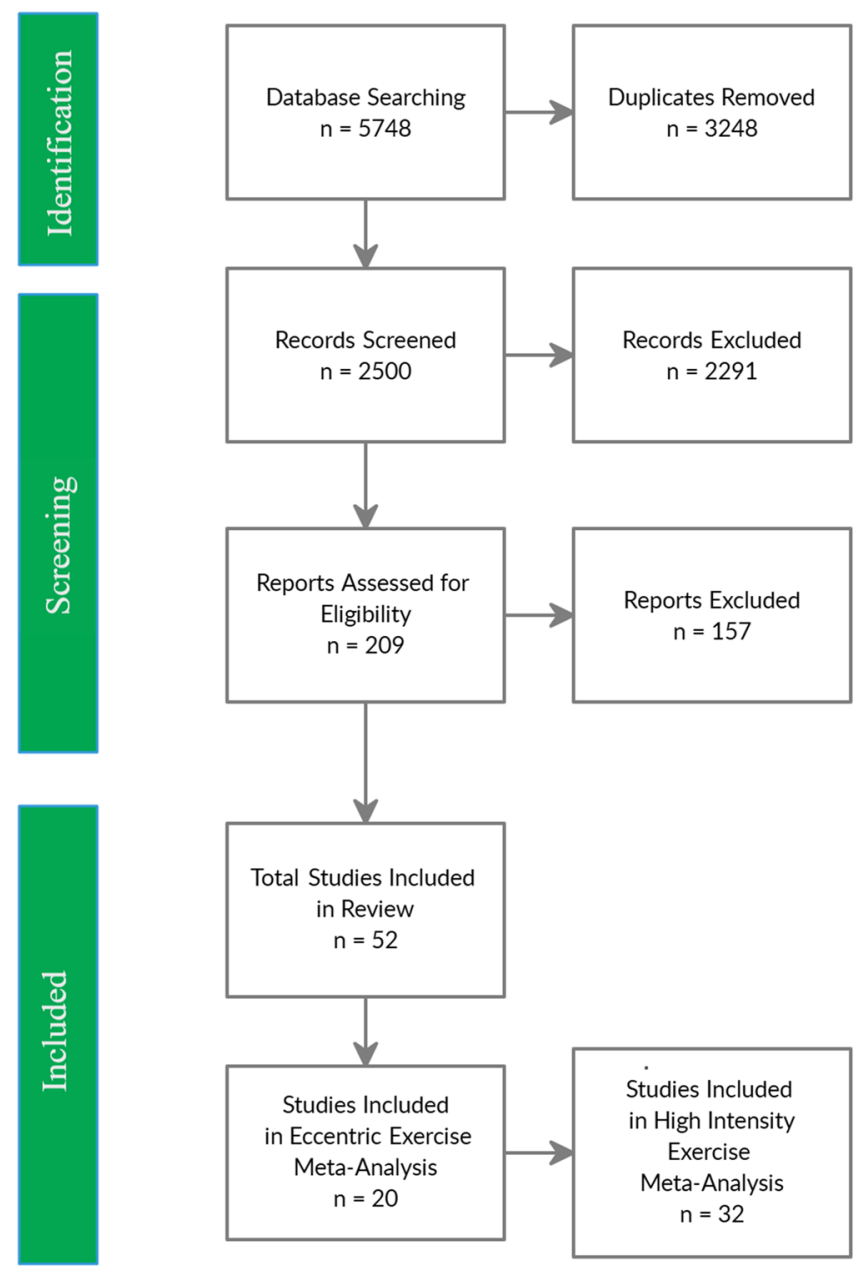

Report Exclusion Reasons - Abstract/Clinical Trial/Thesis $n=37$ - Outcomes other than those mentioned $n=27$ - Studies reported insufficient data $=n=19$

- Participants under $18 n=16$

- Untrained population $n=14$

- Recovery method other than passive recovery $n=14$

Chronic exercise program $n=7$

- Other intervention types $n=7$

- Combined recovery interventions $n=7$

- Study other than RCT $n=3$

- Article not in English $n=2$

- Inconsistent intervention durations $n=2$

- No CWI temp measurement $\mathrm{n}=2$

Fig. 1 PRISMA flowchart for screening of articles. $C W I$ cold-water immersion, $R C T$ randomised controlled trial 


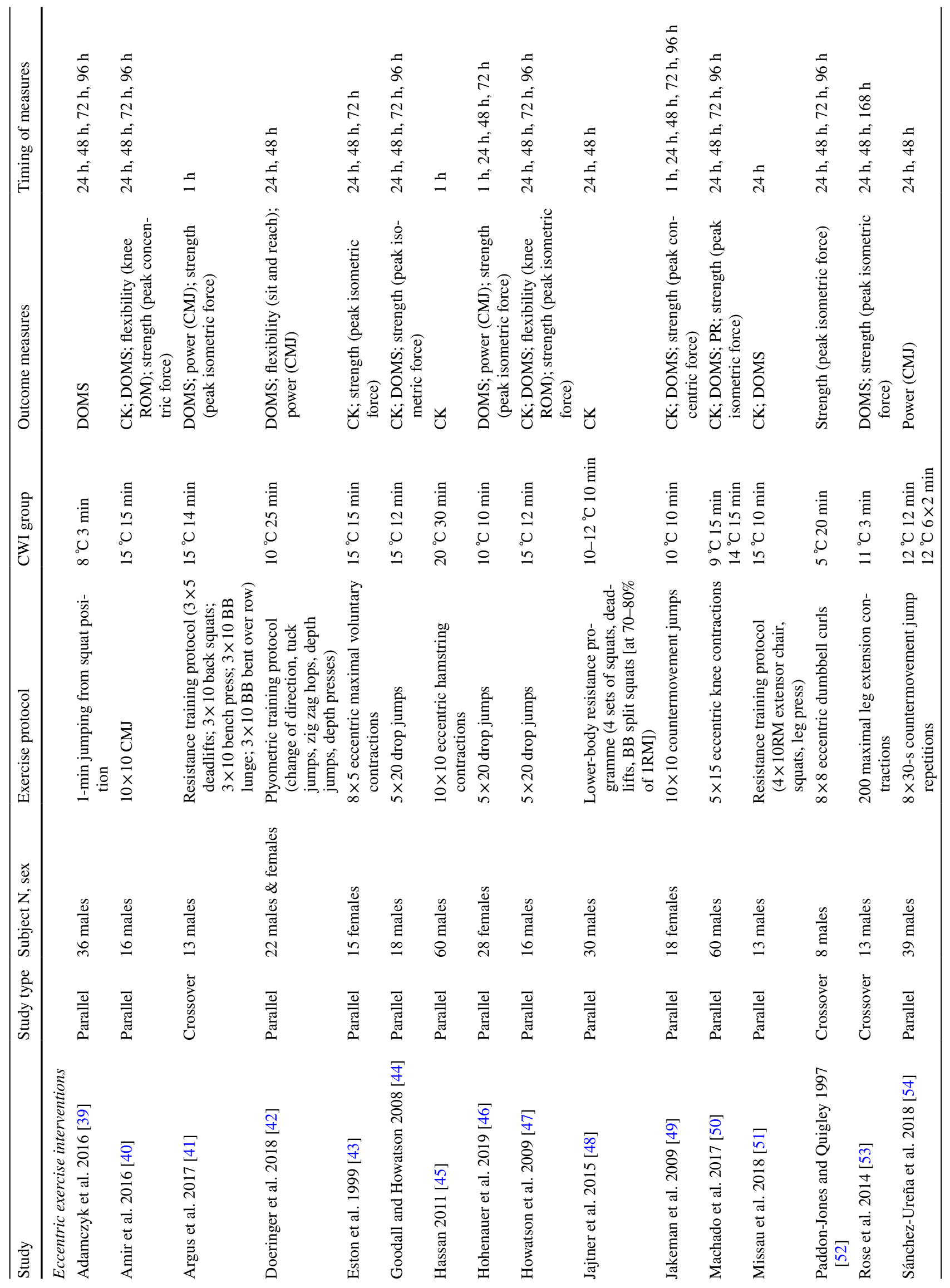




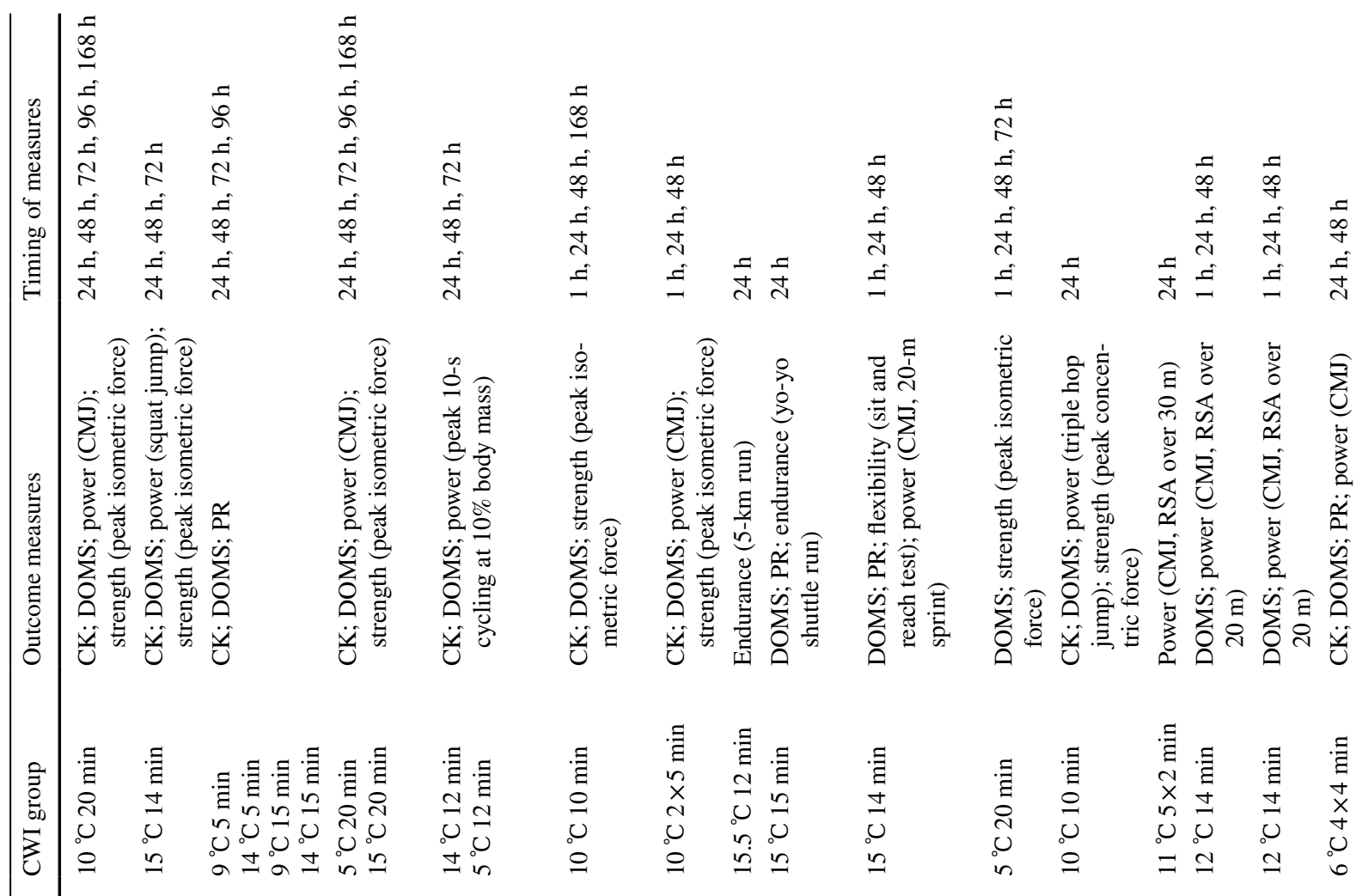

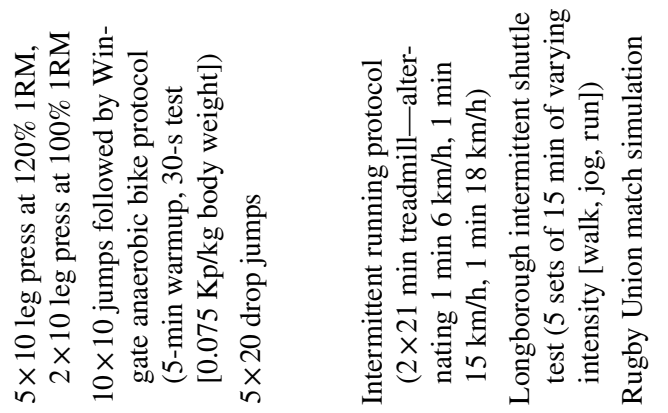

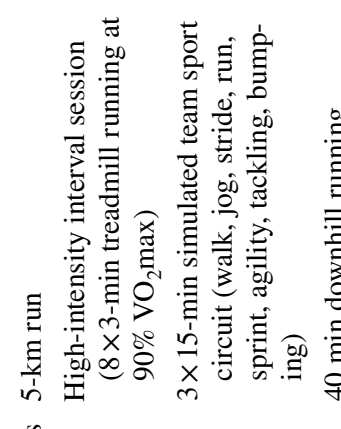

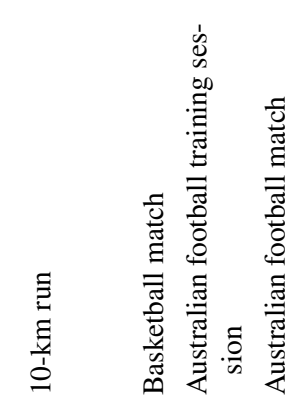

总

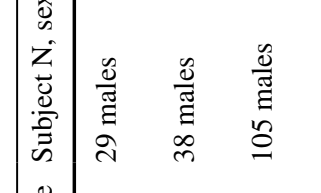

产



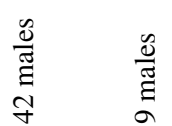

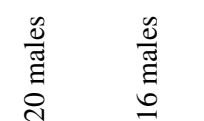

离

喜

等

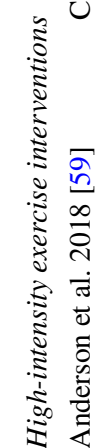

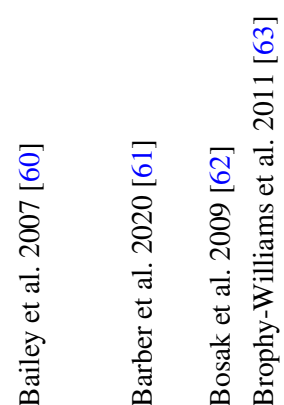
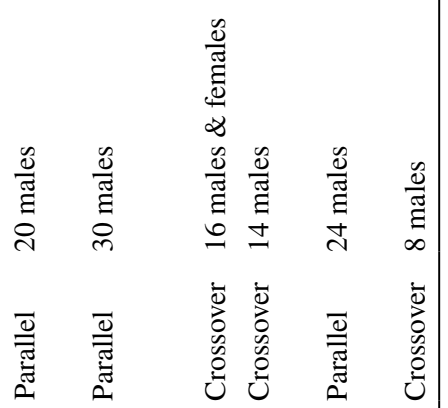

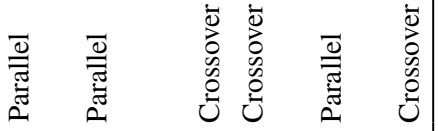




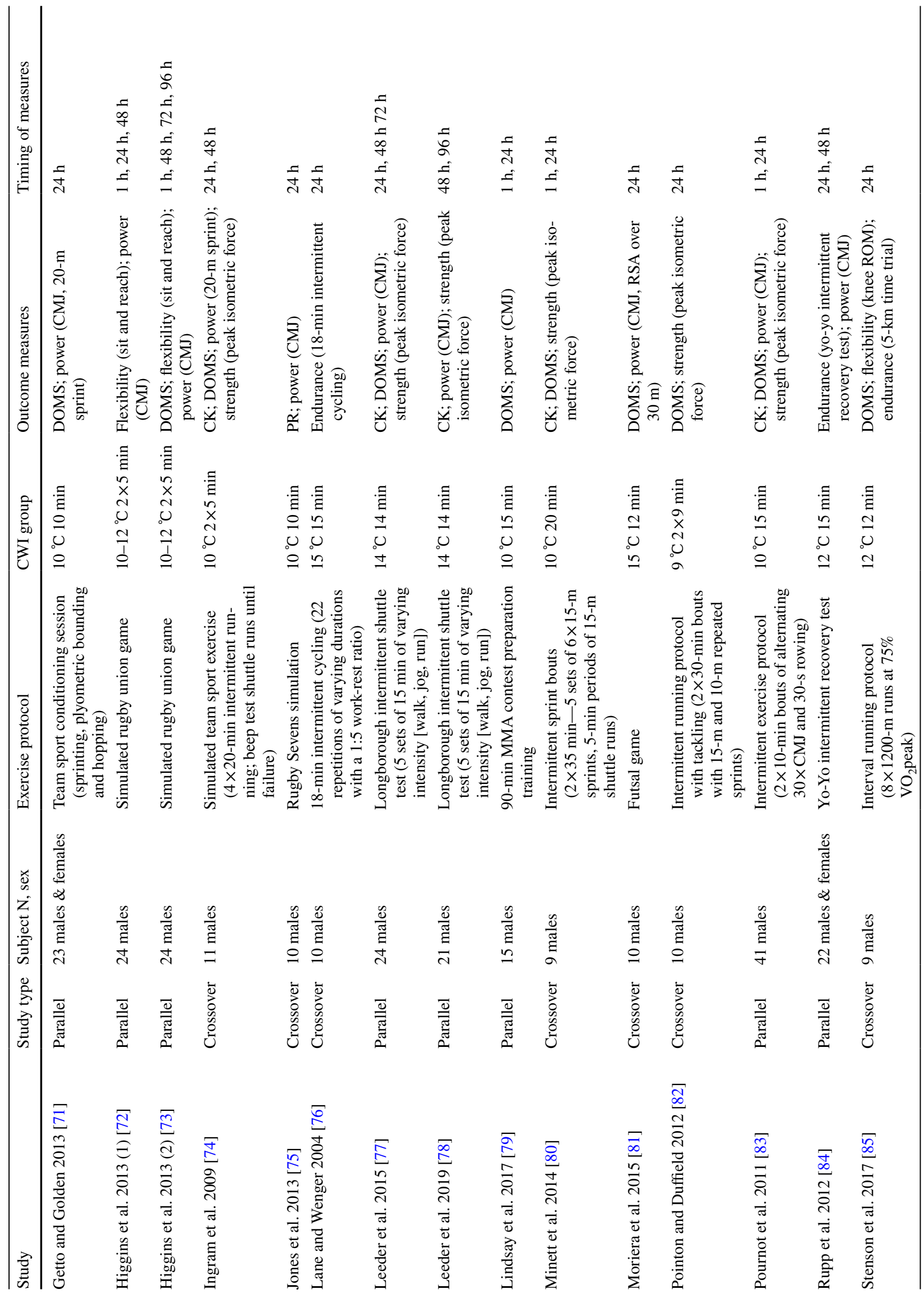




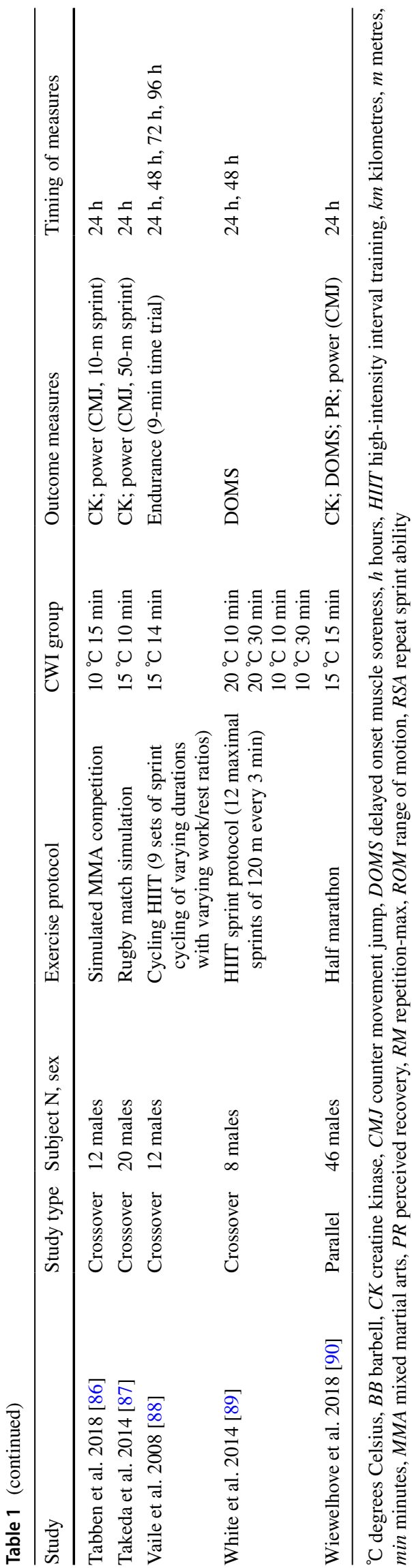

passive recovery at $1 \mathrm{~h}$ (Table $2 ;$ GRADE $=$ moderate $)$ and no effect at $48 \mathrm{~h}$ (Table 2; GRADE $=$ high), $72 \mathrm{~h}$ and $96 \mathrm{~h}$ (Table 2; both timepoints GRADE $=$ moderate). Water temperature and exposure duration had no significant moderating effects at any timepoint.

\subsubsection{The Effects of CWI on Recovery of Strength Performance}

CWI was not effective in promoting the recovery of strength performance $24 \mathrm{~h}$ after eccentric exercise (Fig. 3; Table 2; moderate effect; $p=0.34$; GRADE $=$ moderate). Other timepoints found non-significant very large effects at $1 \mathrm{~h}$ (Table 2; GRADE $=$ low) and moderate effects at 48 and $72 \mathrm{~h}$ (Table 2; both timepoints GRADE $=$ moderate). There were no significant moderating effects of water temperature or exposure duration at any timepoint.

There was no effect of CWI on the recovery of strength performance $24 \mathrm{~h}$ after high-intensity exercise (Fig. 3; Table 2; trivial effect; $p=0.64 ;$ GRADE $=$ moderate) and water temperature and exposure duration did not have significant moderating effects.

\subsubsection{The Effects of CWI on Recovery of Endurance Exercise Performance}

There were no eccentric exercise studies that analysed the effects of CWI on endurance exercise performance.

CWI was not effective in promoting recovery of endurance performance following high-intensity exercise. At 24 and $48 \mathrm{~h}$, there were non-significant trivial effects (Table 2; GRADE $24 \mathrm{~h}=$ very low; GRADE $48 \mathrm{~h}=$ moderate). There was a significant moderating effect of exposure duration at $24 \mathrm{~h}$ where for every 1-min increase in duration, the effect size decreased by 0.15 (95\% CI -0.06 to $-0.24 ; p=0.001$; Fig. 4b).

\subsubsection{The Effects of CWI on Recovery of Flexibility Performance}

CWI was not effective in the recovery of flexibility performance at $48 \mathrm{~h}$ following eccentric exercise (Table 2; small effect; $p=0.48, \mathrm{GRADE}=$ moderate) . There were no other timepoints able to be analysed.

CWI was not effective in promoting the recovery of flexibility performance following high-intensity exercise. Findings at $1 \mathrm{~h}, 24 \mathrm{~h}$ and $48 \mathrm{~h}$ were all non-significant (Table 2; $p>0.05$; GRADE $=$ low).

Due to low study numbers, regression analysis was unable to be completed for flexibility outcomes from any exercise modality at any timepoint. 

the influence of CWI compared with passive recovery $24 \mathrm{~h}$ after exercise on muscular power performance (stratified by exercise intervention modality). $C I$ confidence interval, $C W I$ cold-water immersion, $S M D$ standardised mean difference
Fig. 2 Forest plot illustrating

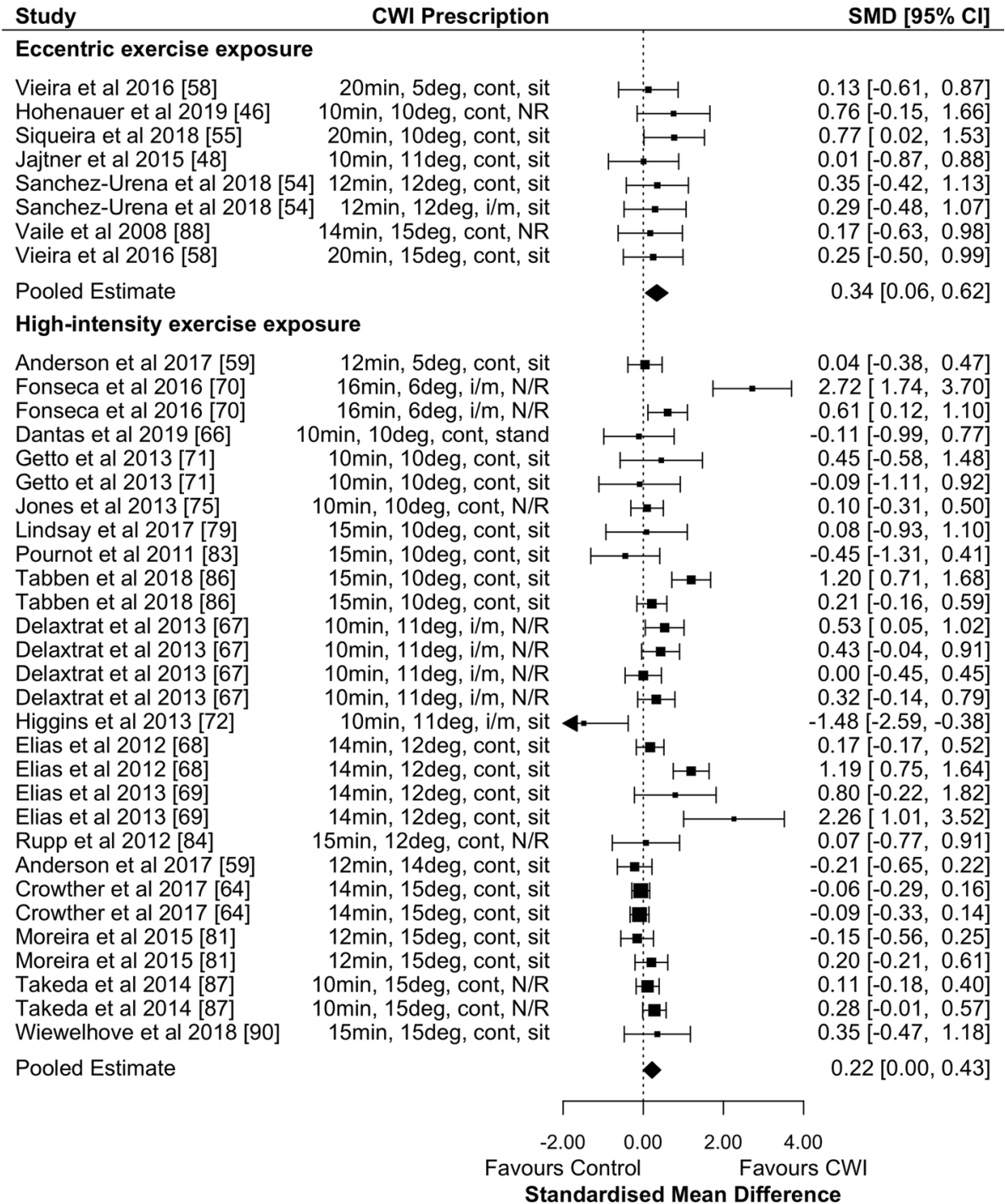

\subsubsection{The Effects of CWI on Recovery from Delayed Onset Muscle Soreness (DOMS)}

CWI was not effective in reducing DOMS $24 \mathrm{~h}$ after eccentric exercise (Fig. 5; Table 2; small effect; $p=0.09$; GRADE $=$ moderate). $\mathrm{CWI}$ was effective at reducing DOMS after eccentric exercise at 48, 72, and $96 \mathrm{~h}$ (Table 2; all small effects; GRADE = high). Water temperature and exposure duration did not have significant moderating effects at any timepoint.

CWI was effective in reducing DOMS $24 \mathrm{~h}$ after highintensity exercise (Fig. 5; Table 2; moderate effect; $p=0.003$; GRADE $=$ moderate $)$. CWI had a moderate effect on reducing DOMS at $1 \mathrm{~h}$ and $48 \mathrm{~h}$ (Table 2; GRADE $=$ moderate). Water temperature and exposure duration did not have significant moderating effects at any timepoint. There was a non-significant trivial effect in favour of passive recovery at
$72 \mathrm{~h}$, but insufficient studies to be meta-analysed at 96 and $168 \mathrm{~h}$ (Table 2).

\subsubsection{The Effects of CWI on Perceived Recovery}

CWI was not effective in increasing feelings of perceived recovery following eccentric exercise at 24 and $48 \mathrm{~h}$ (Table 2; trivial effect; GRADE $=$ high) but did have small beneficial effects at 72 and $96 \mathrm{~h}$ (Table 2; GRADE $=$ high). Water temperature or exposure duration did not have significant moderating effects at any timepoint.

CWI was effective in increasing feelings of perceived recovery $24 \mathrm{~h}$ following high-intensity exercise (Table 2; moderate effect; $p=0.001$; GRADE $=$ moderate). At $48 \mathrm{~h}$, there was a non-significant trivial effect in favour of passive recovery compared with CWI (Table 2; GRADE $=$ high). There were no significant moderating effects of water temperature or exposure duration at any timepoint. 
Table 2 Meta-analysis summary

\begin{tabular}{|c|c|c|c|c|c|c|c|c|c|}
\hline \multirow[t]{2}{*}{ Outcome } & \multicolumn{5}{|c|}{ Summary of findings } & \multicolumn{4}{|c|}{ Quality of evidence synthesis (GRADE) } \\
\hline & $K(k)$ & $N(n)$ & SMD (95\% CI) & $p$-value & $I^{2}(\%)$ & Imprecision & Inconsistency & Risk of bias & Overall quality \\
\hline CK 1 h (ecc) & $2(2)$ & $58(58)$ & $0.85(-0.93$ to 2.63$)$ & 0.349 & 87.5 & -1 & -1 & None & Low \\
\hline CK 1 h (hit) & \multicolumn{9}{|c|}{ Data could not be pooled $(K(k)=1(1))$} \\
\hline CK 24 h (ecc) & $11(16)$ & $373(470)$ & $0.12(-0.31$ to 0.54$)$ & 0.595 & 63.1 & None & -1 & None & Moderate \\
\hline CK 24 h (hit) & $5(6)$ & $105(113)$ & $\begin{array}{c}-0.85(-1.61 \\
\text { to }-0.08)\end{array}$ & 0.030 & 58.6 & None & -1 & None & Moderate \\
\hline CK 48 h (ecc) & $11(16)$ & $373(470)$ & $\begin{array}{l}-0.04(-0.37 \text { to } \\
0.30)\end{array}$ & 0.834 & 50.6 & None & -1 & None & Moderate \\
\hline CK 48 h (hit) & $4(5)$ & $81(89)$ & $\begin{array}{c}-1.36(-2.51 \\
\text { to }-0.20)\end{array}$ & 0.022 & 77.3 & -1 & -1 & None & Low \\
\hline CK 72 h (ecc) & $10(15)$ & $353(450)$ & $\begin{array}{l}-0.15(-0.57 \text { to } \\
0.27)\end{array}$ & 0.494 & 61.5 & None & -1 & None & Moderate \\
\hline CK 72 h (hit) & \multicolumn{9}{|c|}{ Data could not be pooled $(K(k)=1(2))$} \\
\hline CK 96 h (ecc) & $6(10)$ & $243(326)$ & $\begin{array}{l}-0.04(-0.43 \text { to } \\
0.35)\end{array}$ & 0.839 & 39.4 & None & None & None & High \\
\hline CK 96 h (hit) & \multicolumn{9}{|c|}{ Data could not be pooled $(K(k)=1(1))$} \\
\hline CK 168 h (ecc) & $2(3)$ & $71(85)$ & $\begin{array}{c}-0.62(-1.05 \\
\text { to }-0.18)\end{array}$ & 0.006 & 0 & -1 & None & None & Moderate \\
\hline CK 168 h (hit) & \multicolumn{9}{|c|}{ No studies } \\
\hline DOMS $1 \mathrm{~h}(\mathrm{ecc})$ & $4(4)$ & $64(90)$ & $0.00(-1.09$ to 1.10$)$ & 0.998 & 69.4 & -1 & -1 & None & Moderate \\
\hline DOMS 1 h (hit) & $9(10)$ & $149(229)$ & $\begin{array}{l}-1.10(-1.81 \\
\text { to }-0.40)\end{array}$ & 0.002 & 78.1 & None & -1 & None & Moderate \\
\hline DOMS 24 h (ecc) & $13(17)$ & $401(497)$ & $\begin{array}{l}-0.51(-1.10 \text { to } \\
0.09)\end{array}$ & 0.094 & 75.9 & None & -1 & None & Moderate \\
\hline DOMS 24 h (hit) & $20(25)$ & 297 (497) & $\begin{array}{c}-0.89(-1.48 \\
\text { to }-0.29)\end{array}$ & 0.003 & 77.4 & None & -1 & None & Moderate \\
\hline DOMS 48 h (ecc) & $12(16)$ & $375(471)$ & $\begin{array}{c}-0.48(-0.79 \\
\text { to }-0.16)\end{array}$ & 0.003 & 49.1 & None & None & None & High \\
\hline DOMS 48 h (hit) & $11(16)$ & $164(330)$ & $\begin{array}{c}-0.82(-1.57 \\
\text { to }-0.07)\end{array}$ & 0.031 & 83.2 & None & -1 & None & Moderate \\
\hline DOMS 72 h (ecc) & $10(14)$ & $340(423)$ & $\begin{array}{c}-0.55(-0.86 \\
\text { to }-0.23)\end{array}$ & 0.001 & 33.4 & None & None & None & High \\
\hline DOMS 72 h (hit) & $4(6)$ & 69 (104) & $0.09(-0.40$ to 0.59$)$ & 0.718 & 23.9 & -1 & None & None & Moderate \\
\hline DOMS 96 h (ecc) & $8(12)$ & $296(379)$ & $\begin{array}{c}-0.41(-0.62 \\
\text { to }-0.21)\end{array}$ & $<0.001$ & 0 & None & None & None & High \\
\hline DOMS 96 h (hit) & \multicolumn{9}{|c|}{ Data could not be pooled $(K(k)=1(1))$} \\
\hline DOMS 168 h (ecc) & $2(2)$ & $42(55)$ & $\begin{array}{l}-0.78(-1.87 \text { to } \\
0.31)\end{array}$ & 0.160 & 75.0 & -1 & -1 & None & Low \\
\hline DOMS 168 h (hit) & \multicolumn{9}{|c|}{ Data could not be pooled $(K(k)=1(1))$} \\
\hline Power $1 \mathrm{~h}(\mathrm{ecc})$ & $2(2)$ & $33(46)$ & $\begin{array}{l}-0.25(-0.58 \text { to } \\
0.07)\end{array}$ & 0.123 & 9.9 & -1 & None & None & Moderate \\
\hline Power 1 h (hit) & $4(5)$ & $84(174)$ & $\begin{array}{l}-0.22(-0.52 \text { to } \\
0.09)\end{array}$ & 0.160 & 14.3 & -1 & None & None & Moderate \\
\hline Power $24 \mathrm{~h}(\mathrm{ecc})$ & $6(8)$ & $174(201)$ & $0.34(0.06$ to 0.62$)$ & 0.018 & 0 & None & None & None & High \\
\hline Power 24 h (hit) & $17(29)$ & $278(676)$ & $0.22(0.004$ to 0.43$)$ & 0.046 & 74.6 & None & -1 & None & Moderate \\
\hline Power $48 \mathrm{~h}$ (ecc) & $7(10)$ & $196(245)$ & $0.47(0.22$ to 0.73$)$ & $<0.001$ & 0 & None & None & None & High \\
\hline Power 48 h (hit) & $10(14)$ & $163(357)$ & $\begin{array}{l}-0.02(-0.12 \text { to } \\
0.07)\end{array}$ & 0.608 & 4.8 & None & None & None & High \\
\hline Power 72 h (ecc) & $4(5)$ & $115(129)$ & $0.62(0.27$ to 0.98$)$ & 0.001 & 0 & None & None & None & High \\
\hline Power 72 h (hit) & $2(3)$ & $25(52)$ & $\begin{array}{l}-0.01(-0.51 \text { to } \\
0.49)\end{array}$ & 0.969 & 0 & -1 & None & None & Moderate \\
\hline Power 96 h (ecc) & $2(3)$ & $71(85)$ & $0.41(-0.02$ to 0.84$)$ & 0.062 & 0 & -1 & None & None & Moderate \\
\hline Power 96 h (hit) & $2(2)$ & $37(37)$ & $0.05(-0.59$ to 0.70$)$ & 0.873 & 0 & -1 & None & None & Moderate \\
\hline
\end{tabular}


Table 2 (continued)

\begin{tabular}{|c|c|c|c|c|c|c|c|c|c|}
\hline \multirow[t]{2}{*}{ Outcome } & \multicolumn{5}{|c|}{ Summary of findings } & \multicolumn{4}{|c|}{ Quality of evidence synthesis (GRADE) } \\
\hline & $K(k)$ & $N(n)$ & SMD $(95 \%$ CI $)$ & $p$-value & $I^{2}(\%)$ & Imprecision & Inconsistency & Risk of bias & Overall quality \\
\hline Power $168 \mathrm{~h}(\mathrm{ecc})$ & $2(3)$ & $71(85)$ & $0.36(-0.23$ to 0.95$)$ & 0.228 & 0 & -1 & None & None & Moderate \\
\hline Power 168 h (hit) & \multicolumn{9}{|c|}{ Data could not be pooled $(K(k)=1(1))$} \\
\hline Strength $1 \mathrm{~h}(\mathrm{ecc})$ & $2(2)$ & $38(38)$ & $2.31(-4.25$ to 8.88$)$ & 0.490 & 97.2 & -1 & -1 & None & Low \\
\hline Strength $1 \mathrm{~h}$ (hit) & $2(2)$ & $42(42)$ & $\begin{array}{l}-0.11(-0.72 \text { to } \\
0.50)\end{array}$ & 0.720 & 0 & -1 & None & None & Moderate \\
\hline Strength $24 \mathrm{~h}(\mathrm{ecc})$ & $8(10)$ & $224(258)$ & $0.74(-0.78$ to 2.26$)$ & 0.340 & 76.0 & None & -1 & None & Moderate \\
\hline Strength 24 h (hit) & $3(3)$ & $62(62)$ & $0.12(-0.38$ to 0.62$)$ & 0.637 & 0 & -1 & None & None & Moderate \\
\hline Strength $48 \mathrm{~h}(\mathrm{ecc})$ & $8(10)$ & $224(258)$ & $0.84(-0.89$ to 2.57$)$ & 0.339 & 78.9 & None & -1 & None & Moderate \\
\hline Strength 48 h (hit) & \multicolumn{9}{|c|}{ Data could not be pooled $(K(k)=1(1))$} \\
\hline Strength $72 \mathrm{~h}(\mathrm{ecc})$ & $8(10)$ & $224(258)$ & $0.64(-0.51$ to 1.79$)$ & 0.276 & 74.9 & None & -1 & None & Moderate \\
\hline Strength 72 h (hit) & \multicolumn{9}{|c|}{ Data could not be pooled $(K(k)=1(1))$} \\
\hline Strength 96 h (ecc) & $5(7)$ & 165 (199) & $\begin{array}{l}-0.30(-0.78 \text { to } \\
0.17)\end{array}$ & 0.212 & 36.8 & None & None & None & High \\
\hline Strength 96 h (hit) & \multicolumn{9}{|c|}{ No studies } \\
\hline Strength $168 \mathrm{~h}(\mathrm{ecc})$ & $2(3)$ & $71(85)$ & $\begin{array}{l}-0.09(-0.51 \text { to } \\
0.34)\end{array}$ & 0.691 & 0 & -1 & None & None & Moderate \\
\hline Strength 168 h (hit) & \multicolumn{9}{|c|}{ No studies } \\
\hline $\begin{array}{l}\text { Perceived recovery } \\
24 \mathrm{~h}(\mathrm{ecc})\end{array}$ & $2(6)$ & $159(242)$ & $0.15(-0.29$ to 0.59$)$ & 0.493 & 0 & None & None & None & High \\
\hline $\begin{array}{l}\text { Perceived recovery } \\
24 \text { h (hit) }\end{array}$ & $5(5)$ & 79 (135) & $0.66(0.29$ to 1.03$)$ & 0.001 & 34.3 & -1 & None & None & Moderate \\
\hline $\begin{array}{l}\text { Perceived recovery } \\
48 \mathrm{~h}(\mathrm{ecc})\end{array}$ & $2(6)$ & $159(242)$ & $0.14(-0.11$ to 0.39$)$ & 0.274 & 0 & None & None & None & High \\
\hline $\begin{array}{l}\text { Perceived recovery } \\
48 \text { h (hit) }\end{array}$ & $2(2)$ & $38(76)$ & $\begin{array}{l}-0.02(-0.38 \text { to } \\
0.34)\end{array}$ & 0.901 & 0 & -1 & None & None & High \\
\hline $\begin{array}{l}\text { Perceived recovery } \\
72 \mathrm{~h}(\mathrm{ecc})\end{array}$ & $2(6)$ & $159(242)$ & $0.55(0.29$ to 0.80$)$ & $<0.001$ & 0 & None & None & None & High \\
\hline $\begin{array}{l}\text { Perceived recovery } \\
72 \mathrm{~h} \text { (hit) }\end{array}$ & \multicolumn{9}{|c|}{ No studies } \\
\hline $\begin{array}{l}\text { Perceived recovery } \\
96 \mathrm{~h}(\mathrm{ecc})\end{array}$ & $2(6)$ & $159(242)$ & $0.38(0.12$ to 0.64$)$ & 0.004 & 2.9 & None & None & None & High \\
\hline $\begin{array}{l}\text { Perceived recovery } \\
96 \text { h (hit) }\end{array}$ & \multicolumn{9}{|c|}{ No studies } \\
\hline Endurance $24 \mathrm{~h}$ (ecc) & \multicolumn{9}{|c|}{ No studies } \\
\hline Endurance 24 h (hit) & $5(5)$ & $51(102)$ & $\begin{array}{l}-0.14(-0.40 \text { to } \\
0.13)\end{array}$ & 0.316 & 73.6 & -1 & -1 & -1 & Very low \\
\hline Endurance $48 \mathrm{~h}$ (ecc) & \multicolumn{9}{|c|}{ No studies } \\
\hline Endurance 48 h (hit) & $2(2)$ & $24(46)$ & $0.12(-0.16$ to 0.40$)$ & 0.400 & 0 & -1 & None & None & Moderate \\
\hline Flexibility $1 \mathrm{~h}(\mathrm{ecc})$ & \multicolumn{9}{|c|}{ No studies } \\
\hline Flexibility 1 h (hit) & $3(3)$ & $62(92)$ & $2.14(-1.77$ to 6.06$)$ & 0.283 & 92.9 & -1 & -1 & None & Low \\
\hline Flexibility 24 h (ecc) & \multicolumn{9}{|c|}{ Data could not be pooled $(K(k)=1(1))$} \\
\hline Flexibility 24 h (hit) & $3(3)$ & $55(94)$ & $1.52(-1.78$ to 4.82$)$ & 0.367 & 92.6 & -1 & -1 & None & Low \\
\hline Flexibility $48 \mathrm{~h}(\mathrm{ecc})$ & $2(2)$ & $38(38)$ & $0.23(-0.41$ to 0.87$)$ & 0.481 & 0 & -1 & None & None & Moderate \\
\hline Flexibility 48 h (hit) & $3(3)$ & $62(92)$ & $2.23(-1.86$ to 6.32$)$ & 0.286 & 93.3 & -1 & -1 & None & Low \\
\hline Flexibility 72 h (ecc) & \multicolumn{9}{|c|}{ Data could not be pooled $(K(k)=1(1))$} \\
\hline Flexibility 72 h (hit) & \multicolumn{9}{|c|}{ Data could not be pooled $(K(k)=1(1))$} \\
\hline Flexibility 96 h (ecc) & \multicolumn{9}{|c|}{ Data could not be pooled $(K(k)=1(1))$} \\
\hline Flexibility 96 h (hit) & \multicolumn{9}{|c|}{ Data could not be pooled $(K(k)=1(1))$} \\
\hline
\end{tabular}

$C K$ creatine kinase, DOMS delayed onset muscle soreness, ecc eccentric exercise, GRADE Grading of Recommendations Assessment, Development and Evaluation, $h$ hour, hit high-intensity exercise, $K(k)$ unique studies (observation points), $N(n)$ unique participants (observation points), SMD standardised mean difference, $95 \%$ CI 95\% confidence interval 
Fig. 3 Forest plot illustrating the influence of CWI compared with passive recovery $24 \mathrm{~h}$ after exercise on strength performance (stratified by exercise intervention modality). CI confidence interval, $C W I$ cold-water immersion, $S M D$ standardised mean difference
Fig. 4 Meta-regression plots illustrating the influence of CWI duration and temperature on endurance performance and removal of serum CK from the blood at $24 \mathrm{~h}$ and $48 \mathrm{~h}$ after exercise. a Effect of time at $24 \mathrm{~h}$ after exercise; $\mathbf{b}$ effect of time at $48 \mathrm{~h}$ after exercise; $\mathbf{c}$ effect of temperature at $24 \mathrm{~h}$ after exercise; $\mathbf{d}$ effect of temperature at $48 \mathrm{~h}$ after exercise. $C K$ creatine kinase, $C W I$ cold-water immersion, $S M D$ standardised mean difference

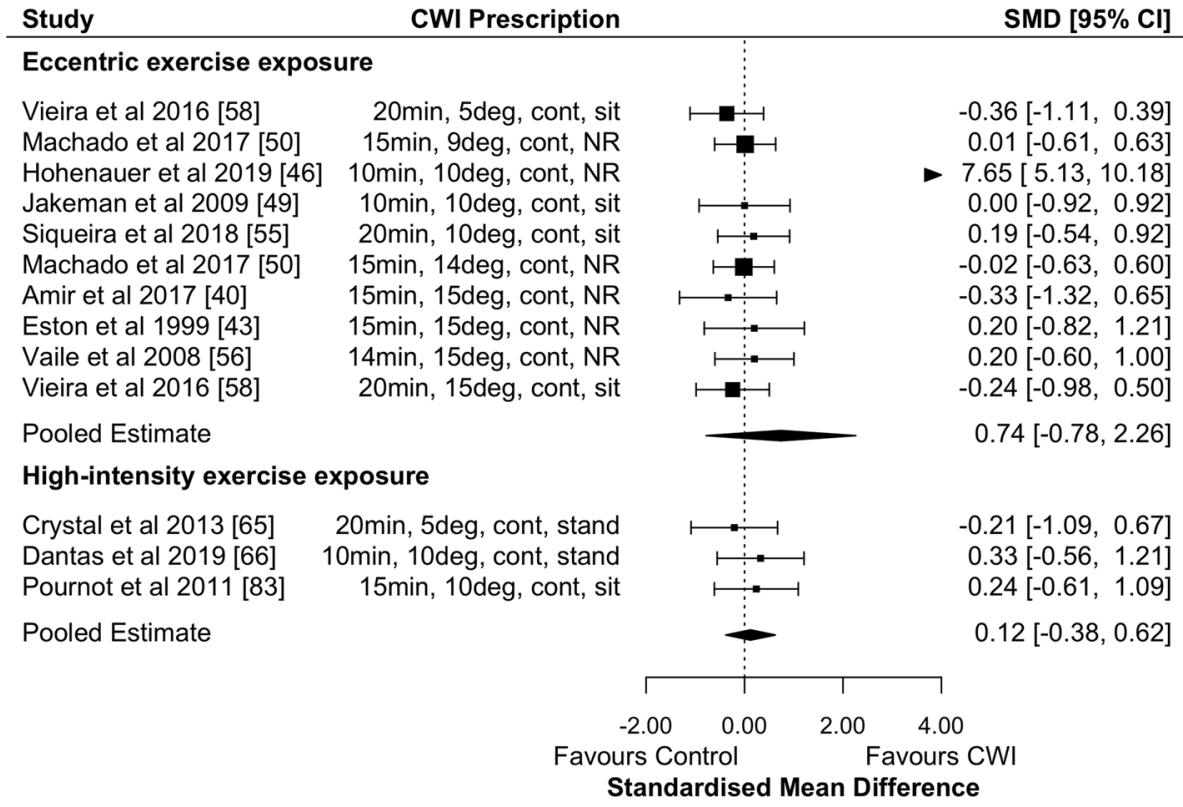

a

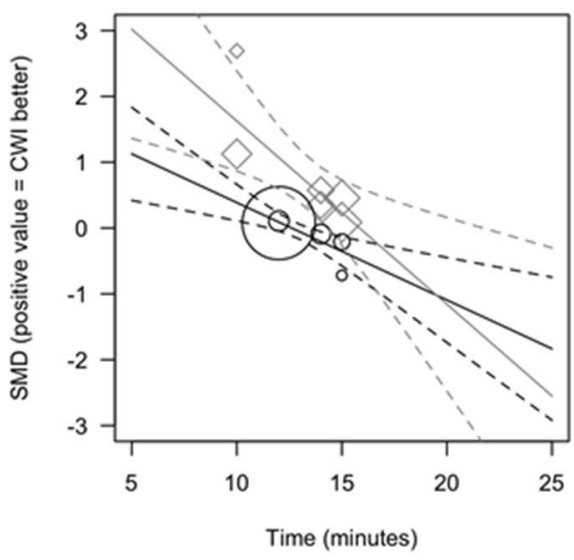

C

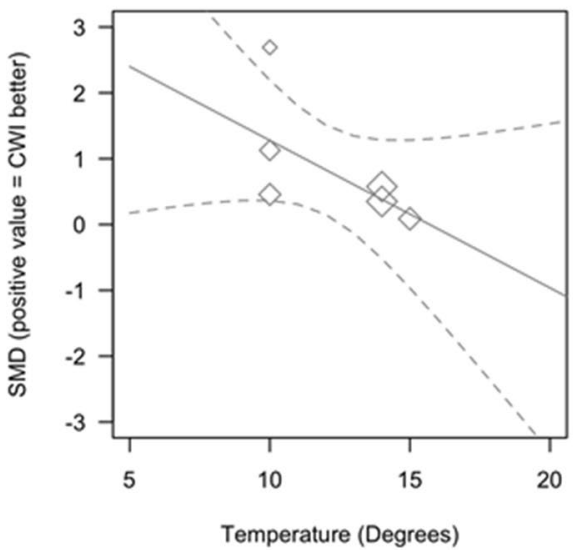

b

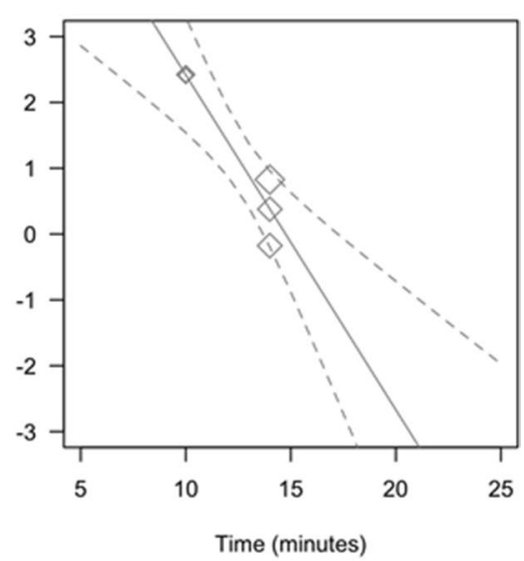

d

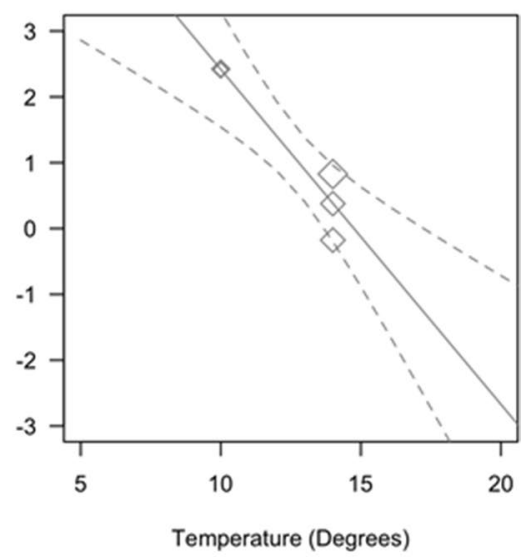


Fig. 5 Forest plot illustrating the influence of CWI compared with passive recovery $24 \mathrm{~h}$ after exercise on muscle soreness (stratified by exercise intervention modality). $C I$ confidence interval, $C W I$ cold-water immersion, $S M D$ standardised mean difference

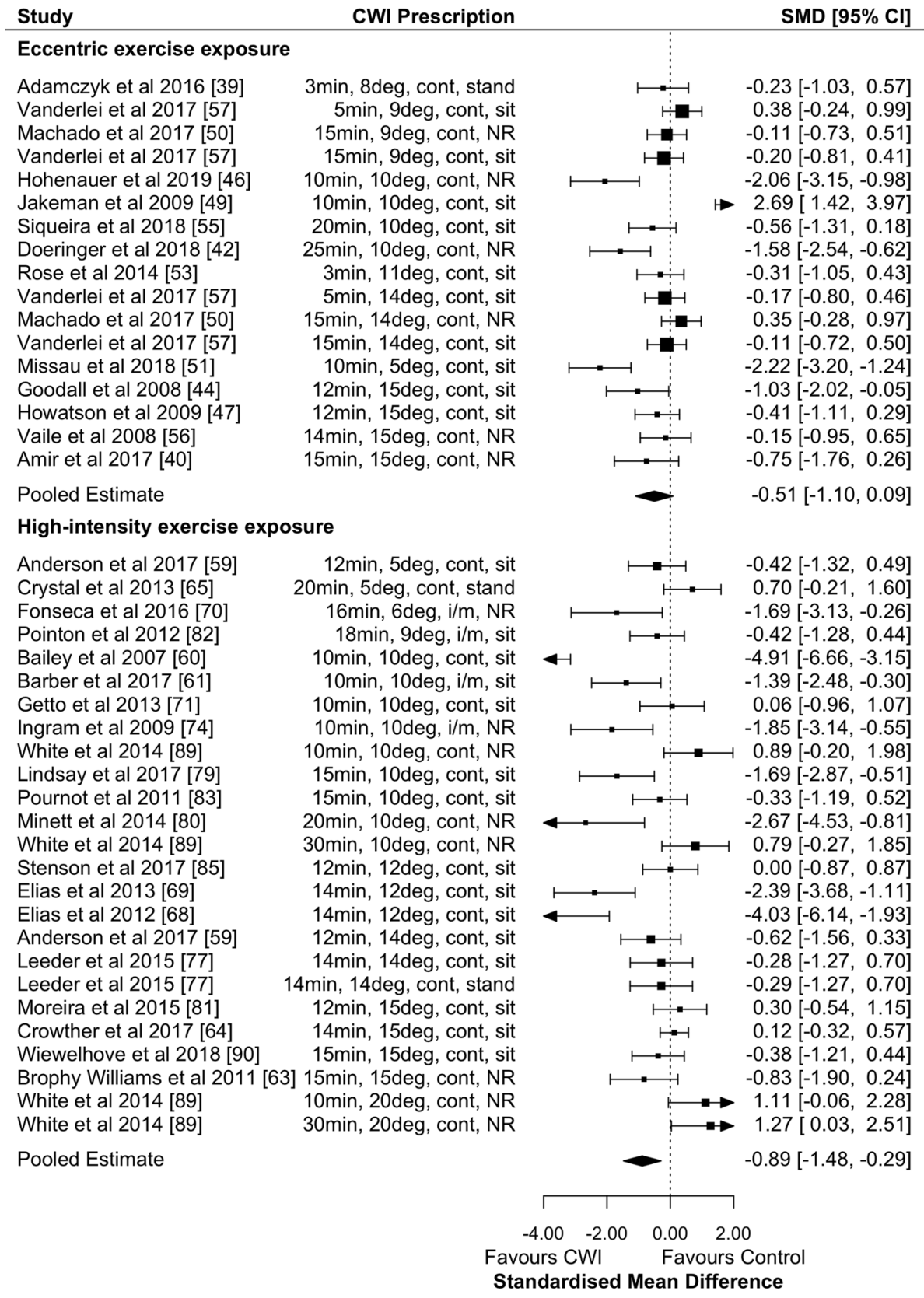

\subsubsection{The Effects of CWI on Recovery of Creatine Kinase}

CWI was not effective in reducing circulating CK $24 \mathrm{~h}$ after eccentric exercise (Fig. 6; trivial effect; $p=0.60$; GRADE $=$ moderate). The only significant timepoint where CK was reduced after eccentric exercise using CWI was at 168 h (i.e. 7 days) (Table 2; GRADE $=$ moderate). Other timepoints showed non-significant trivial effects in favour of CWI (Table 2; GRADE $48 \mathrm{~h}$ and $72 \mathrm{~h}=$ moderate; GRADE $96 \mathrm{~h}=$ high), except for $1 \mathrm{~h}$ which showed large non-significant effects in favour of passive recovery (Table 2; GRADE $1 \mathrm{~h}=$ low). There were no significant moderating effects of water temperature or exposure duration at any timepoint.
CWI was effective in reducing circulating CK $24 \mathrm{~h}$ after high-intensity exercise (Fig. 6; Table 2; moderate effect; $p=0.03$; GRADE $=$ moderate). There was a significant exposure duration moderating effect at $24 \mathrm{~h}$ whereby for every 1-min increase in exposure duration, the effect size decreased by 0.28 (95\% CI -0.09 to $-0.47 ; p=0.004$; Fig. 4a). The only other timepoint that showed significant results after high-intensity exercise was at $48 \mathrm{~h}$ (Table 2; GRADE $=$ moderate). There were significant moderating effects at $48 \mathrm{~h}$ of both water temperature and exposure duration, whereby for every 1-min increase in duration (Fig. 4b) and every $1{ }^{\circ} \mathrm{C}$ increase in temperature (Fig. 4d), the effect size for both duration and temperature decreased by 0.51 
Fig. 6 Forest plot illustrating the influence of CWI compared with passive recovery $24 \mathrm{~h}$ (stratified by exercise intervention modality). $C I$ confidence interval, $C K$ creatine kinase, $C W I$ cold-water immersion, $S M D$ standardised mean difference after exercise on removal of CK

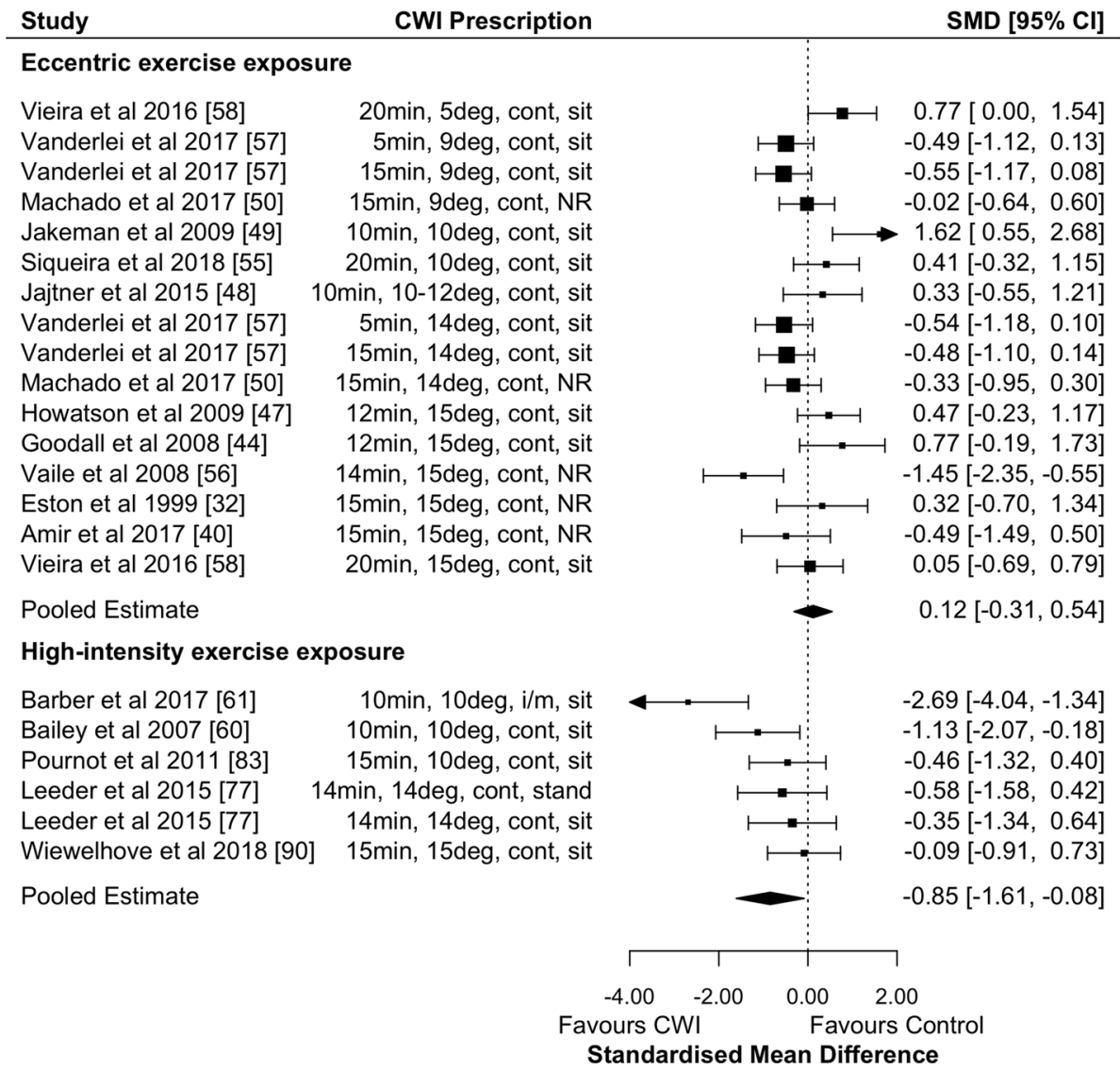

(95\% CI -0.25 to $-0.77 ; p<0.001)$. There were insufficient studies to conduct meta-analyses for the other timepoints.

\section{Discussion}

The aim of the present review was to examine the efficacy of CWI for promoting the recovery of numerous physiological, perceptual and athletic performance variables, as well as attempt to identify dose-response relationships between CWI temperature and/or duration with outcome measures through meta-regression. Overall, results were mixed, but some key findings were evident. CWI was effective at positively influencing power performance for both eccentric and high-intensity exercise. CWI was also effective at reducing $\mathrm{CK}$ concentrations as well as reducing DOMS and increasing perceived recovery from both eccentric and high-intensity exercise. CWI dose-response relationships involving water temperature and/or exposure duration were evident after high-intensity exercise and indicated that shorter time and lower temperatures were related to the largest effects on serum CK concentrations (duration and temperature dose effects), and endurance performance (duration dose effects only).
To the authors' knowledge, this is the first review to compare CWI and passive recovery and their effects on recovery of physiological, perceptual and athletic performance measures at specific timepoints following differing exercise interventions in athletic populations, making it it most relevant to the individuals that use this recovery method the most. Despite using a narrower search strategy than previous reviews, this review identified the greatest number of studies included in CWI meta-analyses. This is also the first review to use meta-regression to determine significant dose-response relationships between water temperature and/or exposure durations, and various outcome measures. Furthermore, this is also the first review to account for methodological variations within parallel and crossover study designs, which is important because this research area includes both parallel and crossover studies.

\subsection{Effect of CWI on Performance Measures}

A variety of performance measures were utilised to allow practitioners to decide whether CWI is an appropriate method of recovery to influence performance (Fig. 7). These measures included muscular strength, flexibility, muscular power (such as sprint performance, jump performance and anaerobic power performance) and endurance performance. 


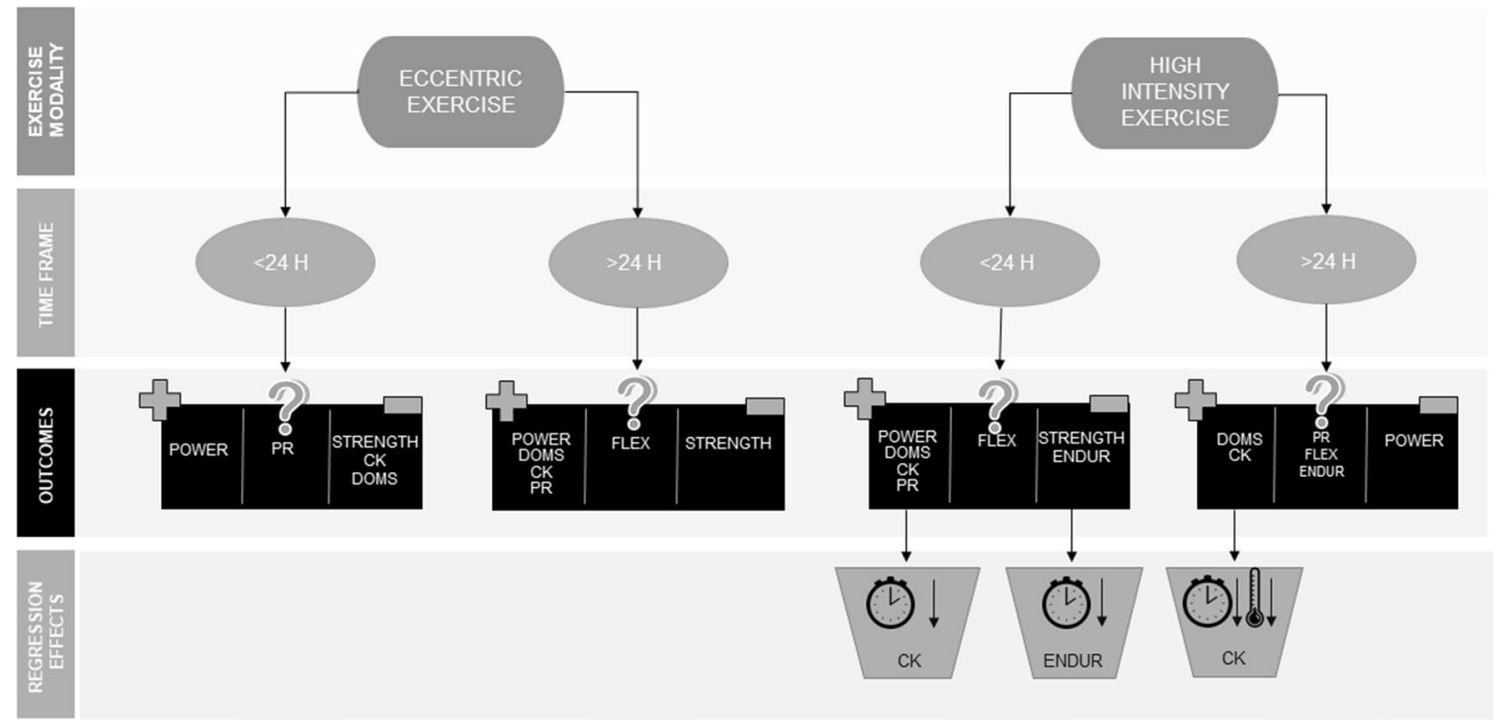

Fig. 7 Summary of review outcomes presented to allow practitioners easy interpretation. $C K$ creatine kinase, DOMS delayed onset muscle soreness, Endur endurance, Flex flexibility, $H$ hours, $P R$ perceived recovery

There was some variability in the influence of CWI on the outcome measures between eccentric and high-intensity exercise, potentially due to the different nature of exercise [8]. The variability of the influence of CWI on the outcome measures may also be accounted for by the training status of the participants; physically active participants may have less muscle damage induced through exercise than their untrained counterparts [91].

\subsubsection{Following Eccentric Exercise}

Pooled results from eccentric exercise studies show that muscular power performance but not strength was mostly likely to be significantly positively influenced using CWI after eccentric exercise. This allows some hypotheses to be drawn in terms of how CWI influences muscular physiology. There is the potential that CWI may specifically impact powerful dynamic movements rather than static strength [8]. Most studies (6/8) included in the recovery of muscular strength analysis used isometric strength testing $[43,46,50,55,56,58]$, and as isometric strength is slower to develop force than dynamic power activities because of the lack of a stretch-shortening cycle, so force production is more reliant on the stiffness of the musculo-tendinous system [92]. Research reports that increased musculo-tendinous stiffness increases isometric force, whereas reduced musculo-tendinous stiffness increases the performance of movements reliant on the stretch-shortening cycle [93]. Considering that dynamic power movements are reliant on the stretch-shortening cycle producing force during eccentric and concentric phases of movement, there is the possibility that CWI reduces musculo-tendinous stiffness within the body, explaining why dynamic muscular power is more positively influenced by CWI than isometric muscular strength. However, it should be noted that some strength outcomes had positive effect sizes but were non-significant due to study variability. The non-significant positive results for strength recovery contrasts with a previous review that concluded that cooling of the neuromuscular system inhibits isometric muscular strength [94]. In addition, cooling of the neuromuscular system reduces central nervous system fatigue [95], which could be why dynamic muscular performance has been significantly improved following CWI and strength results were non-significant (the difference could be the number of studies that used static strength (isometric measures) compared with dynamic strength (concentric or eccentric measures).

CWI did not influence flexibility; however, the limited number of studies $(<2)$ makes it difficult to draw definitive conclusions.

There were no eccentric studies that examined the effect of CWI on endurance performance.

\subsubsection{Following High-Intensity Exercise}

Pooled effects from high-intensity exercise interventions showed that muscular power but not muscular strength was significantly enhanced $24 \mathrm{~h}$ after exercise. This is partially consistent with data from previous eccentric exercise literature, showing that dynamic power activities may be most favourably influenced by CWI reducing musculo-tendinous stiffness $[92,93]$; however, this was only evidenced at $24 \mathrm{~h}$ post-exercise. There were only three studies (two of which used isometric testing [65, 
83]) investigating the recovery of muscular strength following high-intensity exercise that found a non-significant trivial effect in favour of passive recovery $1 \mathrm{~h}$ and $24 \mathrm{~h}$ after exercise. This is in line with the findings for post-eccentric exercise, which could further indicate the influence of CWI on musculo-tendinous stiffness.

The null results shown for the recovery of endurance performance $24 \mathrm{~h}$ after CWI may be the result of the differences in the exercise protocols performed prior to CWI and subsequent performance testing. The studies that performed steady state aerobic protocols prior to CWI found positive effects of CWI during their steady state aerobic performance tests (i.e. 5-km runs) [62, 85]. However, studies that performed more anaerobic-based exercise protocols (i.e. intermittent repeat sprint ability) prior to CWI and endurance performance testing did not find in favour of CWI $[63,76,88]$. It is possible that the vasoconstriction induced by CWI redirects blood flow to the core and increases central blood volume [96]. This increase in central blood volume would lead to increases in stroke volume and cardiac output [97], which would increase aerobic performance. However, the null results for studies using anaerobic-based, repeat-effort exercise protocols prior to CWI could indicate that the glycolytic metabolic by-products increased acidity (and therefore muscle damage) in the muscle [98] to levels greater than what could be effectively cleared using hydrostatic pressure and vasoconstriction seen during CWI [95], resulting in decreased performance, regardless of the positive effects on cardiac dynamics. Further research is required to determine the potential of this hypothesis.

Results from the meta-regression show that shorter duration CWI ( $12 \mathrm{~min}$ ) may positively influence endurance performance more than longer durations (14-15 min) $24 \mathrm{~h}$ after exercise. It is also possible that the temperature of the immersions influenced the null results seen, as lower immersion temperatures result in decreased muscle temperature which leads to decreased blood flow, swelling and oedema [99]; all but one study used a temperature of $15{ }^{\circ} \mathrm{C}$, and meta-regression was therefore not possible due to the lack of variation. The very low GRADE rating for this timepoint indicates the high level of variability amongst the studies.

The limited number of studies assessing flexibility $(<3)$ makes it difficult to draw definitive conclusions from the data. The low GRADE rating evidences the variability within the data at all timepoints.

\subsection{Effect of CWI on Perceptual Measures}

Delayed onset muscle soreness (DOMS) can range from muscle tenderness to severe debilitating pain that can impact subsequent athletic performance until the symptoms ease [100]. Perceived recovery indicates an athlete's impression of how ready they feel for the next activity bout, with adequate recovery opportunities needed to balance the stress state to maintain or increase performance capacity [101]. There is the potential that perceptual measures could be influenced by an athlete's belief in the treatment's efficacy, which may alter their pain perception post-stimulus and provide a placebo effect [102].

\subsubsection{Following Eccentric Exercise}

Pooled results from eccentric exercise showed that CWI did not have significant beneficial effects on DOMS and perceived recovery until $48 \mathrm{~h}$ after exercise. The delayed onset of peak muscle soreness could explain why CWI did not show significant effects on recovery until after $48 \mathrm{~h}$ as athletes may not have experienced significant soreness until this point [103]. Feelings of perceived recovery peaked at the same timepoints, indicating a relationship between diminished feelings of muscle soreness due to CWI and greater feelings of recovery. It is not possible to discount possible placebo effects influencing study results as it is not possible to blind participants to the treatment [104].

\subsubsection{Following High-Intensity Exercise}

Pooled results from high-intensity exercise interventions demonstrated that CWI had an immediate effect on reducing DOMS and increasing feelings of recovery with significant moderate to large effects evident between 1 and $48 \mathrm{~h}$ after exercise. High-intensity exercise has been found to induce high levels of muscle soreness regardless of training status [105], and the decrease in inflammation and oedema induced by the hydrostatic pressure of CWI combined with the analgesic effects of cooling have been shown to reduce DOMS and increase perceived recovery [6, 95]. However, as seen in the eccentric exercise studies, it is not possible to discount possible placebo effects influencing study results as it is impossible to blind participants to the treatment [104].

\subsection{Effect of CWI on Creatine Kinase}

Creatine kinase is a commonly used blood-borne biomarker that indirectly implies muscle damage following strenuous exercise [106]. Serum CK measures represent relative amounts of CK released, the degree of enzyme activity of the released CK and the rate of clearance from the serum [107]. The variation between increases in CK following exercise suggests that the appearance of CK may not be entirely representative of muscle cell damage [108] and could be impacted by sex, ethnicity and age [109]. 


\subsubsection{Following Eccentric Exercise}

Pooled results from eccentric exercise protocols showed that CWI did not influence the removal of CK from the blood at any timepoint, except for $168 \mathrm{~h}$ after exercise. Research has reported that the greatest rise in CK concentration occurs following moderate to high intensity eccentric exercise with multiple exercises and multiple sets, which encourages athletes to exercise to contractile failure $[110,111]$. The studies included in the analysis rarely followed protocols where athletes exercised to failure and only used a single exercise rather than following a protocol that used multiple modalities of exercise that target the same muscle groups (i.e. incorporating push and pull exercises into 'supersets'). It is therefore possible that the single exercise protocols used may not have been sufficiently intense to produce significant rises in CK when comparing CWI with passive recovery. This follows a trend identified by Callegari et al. [110], who reported that less intense eccentric exercise resulted in smaller increases in CK levels in the blood $24 \mathrm{~h}$ after exercise following passive recovery. There is also the possibility that the muscle group subjected to exercise is a factor in the CK response to exercise; research reports that upper body eccentric exercise produces more $\mathrm{CK}$ than lower body eccentric exercise [112], possibly due to the training status of the participant and the repeat bout effect where participants are used to performing lower body eccentric movements (i.e. jumping, squatting) in everyday life and physical training [113]. The lone significant result found at $168 \mathrm{~h}$ after exercise shows that CWI has the potential to reduce CK; the two studies $[55,58]$ (with three observation points) found that significantly reducing CK levels may take 7 days following eccentric exercise.

\subsubsection{Following High-Intensity Exercise}

Pooled results from high-intensity exercise interventions showed that using CWI resulted in significant lower CK concentrations at 24 and $48 \mathrm{~h}$ after exercise. The studies included in this analysis used similar protocols that included high-intensity running as well as team sport simulations that place high levels of stress on the lower limbs. It should be noted that high-intensity exercise is more representative of athletic training when compared with resistance-based exercise. High-intensity running-based exercise results in the greatest rise in CK levels after exercise when compared with lower intensity running [110]; the eccentric nature of running combined with high volumes of running may increase muscle damage (and therefore CK levels) in the lower limbs due to the stretch-shortening cycle that occurs within the muscles [114]. The dose-response relationship for water temperature and immersion duration at $48 \mathrm{~h}$ where a lower temperature may be more effective at shorter durations may be explained by the colder temperatures reducing the efflux of CK from the muscles to the extracellular space via reduced vessel wall permeability [43] as well as reduced inflammation resulting in less secondary tissue damage [4].

\subsection{Limitations and Future Research}

This review has limitations that should be considered when interpreting the findings. The majority of studies used only isometric testing as a measure of strength, so it is not clear if other measures of strength (i.e. one-repetition maximum concentric or eccentric contractions) may be more positively influenced by CWI. Very few studies investigated the effects of CWI on endurance and flexibility performance, and therefore conclusions based on these variables should be interpreted with caution. This review identified only four studies that used female-only cohorts, compared with 44 studies that used male-only cohorts and six studies that used a mixed cohort. As a result, findings from this review might be more reflective of male rather than female responses to CWI.

It is important to highlight the limitations identified through risk of bias. Blinding of participants and researchers is not possible due to the nature of the treatments, which could enhance the potential of the placebo effect of CWI. The limited randomisation of treatment groups could also influence the results, especially in parallel studies where participants are only undertaking one treatment. Incorporating a CWI placebo in addition to the treatment groups may limit the influence of the placebo effect.

Further research should be conducted using female-only cohorts to address the current sex bias in CWI literature; this would address differences in physiology between males and females and enable the identification of appropriate recovery protocols for female athletes. In addition, future research could elucidate whether inter-individual differences (i.e. body morphology, fat and lean body mass) influence the effectiveness of CWI as a recovery protocol. Also, studies investigating the effects of CWI on the recovery of muscular strength should consider various measures of strength rather than isometric testing only to better discern the efficacy of CWI for recovering muscular strength. Given the positive relationships identified in this review, more studies identifying the effects of CWI after high-intensity exercise are needed to further evaluate the effects on endurance performance and flexibility. In addition, evaluations assessing whether participants believing that CWI will aid their recovery influences outcomes should be performed to identify potential belief and placebo effects.

\section{Conclusions}

The present systematic review and meta-analyses identified 52 randomised controlled studies investigating the effect of CWI on the recovery of physiological, perceptual 
and performance outcomes. The findings indicate several benefits of using CWI as a recovery intervention, particularly following high-intensity exercise. A reduction in DOMS and improvements in perceived recovery for both eccentric and high-intensity exercise were observed after CWI. CWI is more likely to positively influence dynamic power movements rather than static strength following both eccentric and high-intensity exercise. Dose-response relationships emerged for several variables indicating that lower durations and temperatures may improve the efficacy of CWI if used after high-intensity exercise.

Supplementary Information The online version contains supplementary material available at https://doi.org/10.1007/s40279-022-01644-9.

Acknowledgements The authors would like to thank Academic Librarian Sarah McQuillen for her assistance in putting together the search strategy.

Data availability statement The datasets generated during and/or analysed during the current systematic review are available in the Online Supplements 2-8, see ESM.

\section{Declarations}

Funding Open Access funding enabled and organized by CAUL and its Member Institutions. Emma Moore is supported by a Research Training Program (Domestic) Scholarship from the Australian Commonwealth Department of Education and Training.

Conflict of interest Emma Moore, Joel T. Fuller, Sienna Saunders, Shona L. Halson, James R. Broatch and Clint R. Bellenger declare that they have no conflict of interest. Jonathan D. Buckley is a recipient of a grant from the Norwood Football Club to evaluate effects of cold-water immersion on recovery of athletic performance. Norwood Football Club had no involvement with the current manuscript.

Author contributions Emma Moore, Jonathan D. Buckley, Shona L. Halson, James R. Broatch and Clint R. Bellenger contributed to the design of the review and completion of the search strategy. Emma Moore and Sienna Saunders completed data screening and data extraction. Joel T. Fuller was responsible for the meta-analysis. Emma Moore drafted the manuscript. All authors edited and revised the manuscript and approved the final version of the manuscript.

Open Access This article is licensed under a Creative Commons Attribution 4.0 International License, which permits use, sharing, adaptation, distribution and reproduction in any medium or format, as long as you give appropriate credit to the original author(s) and the source, provide a link to the Creative Commons licence, and indicate if changes were made. The images or other third party material in this article are included in the article's Creative Commons licence, unless indicated otherwise in a credit line to the material. If material is not included in the article's Creative Commons licence and your intended use is not permitted by statutory regulation or exceeds the permitted use, you will need to obtain permission directly from the copyright holder. To view a copy of this licence, visit http://creativecommons.org/licenses/by/4.0/.

\section{References}

1. Mujika I, Halson S, Burke LM, Balagué G, Farrow D. An integrated, multifactorial approach to periodization for optimal performance in individual and team sports. Int J Sports Physiol Perform. 2018;13(5):538-61.

2. Mujika I. The influence of training characteristics and tapering on the adaptation in highly trained individuals: a review. Int J Sports Med. 1998;19(7):439-46.

3. Meeusen R, Duclos M, Foster C, Fry A, Gleeson M, Nieman D, et al. Prevention, diagnosis, and treatment of the overtraining syndrome: joint consensus statement of the European College of Sport Science and the American College of Sports Medicine. Med Sci Sports Exerc. 2013;45(1):186-205.

4. Bleakley CM, Davison GW. What is the biochemical and physiological rationale for using cold-water immersion in sports recovery? A systematic review. Br J Sports Med. 2010;44(3):179-87.

5. Crowther F, Sealey R, Crowe M, Edwards A, Halson S. Team sport athletes' perceptions and use of recovery strategies: a mixed-methods survey study. BMC Sports Sci Med Rehab. 2017;9(1):6.

6. Wilcock IM, Cronin JB, Hing WA. Physiological response to water immersion. Sports Med. 2006;36(9):747-65.

7. Tipton M, Collier N, Massey H, Corbett J, Harper M. Cold water immersion: kill or cure? Exp Physiol. 2017;102(11):1335-55.

8. Leeder J, Gissane C, van Someren K, Gregson W, Howatson G. Cold water immersion and recovery from strenuous exercise: a meta-analysis. Br J Sports Med. 2011;46(4):233-40.

9. Machado AF, Ferreira PH, Micheletti JK, de Almeida AC, Lemes ÍR, Vanderlei FM, et al. Can water temperature and immersion time influence the effect of cold water immersion on muscle soreness? A systematic review and meta-analysis. Sports Med. 2016;46(4):503-14.

10. Poppendieck W, Faude O, Wegmann M, Meyer T. Cooling and performance recovery of trained athletes: a meta-analytical review. Int J Sports Physiol Perform. 2013;8(3):227-42.

11. Hohenauer E, Taeymans J, Baeyens J-P, Clarys P, Clijsen R. The effect of post-exercise cryotherapy on recovery characteristics: a systematic review and meta-analysis. PLoS ONE. 2015;10(9):1-22.

12. Elbourne DR, Altman DG, Higgins JP, Curtin F, Worthington HV, Vail A. Meta-analyses involving cross-over trials: methodological issues. Int J Epidemiol. 2002;31(1):140-9.

13. Thompson SG, Higgins JP. How should meta-regression analyses be undertaken and interpreted? Stat Med. 2002;21(11):1559-73.

14. Baker W, Michael White C, Cappelleri J, Kluger J, Coleman C. Understanding heterogeneity in meta-analysis: the role of metaregression. Int J Clin Pract. 2009;63(10):1426-34.

15. GRADE Working Group. Grading quality of evidence and strength of recommendations. BMJ. 2004;328(7454):1490-7.

16. Page MJ, McKenzie JE, Bossuyt PM, Boutron I, Hoffmann TC, Mulrow CD, et al. The PRISMA 2020 statement: an updated guideline for reporting systematic reviews. BMJ. 2021;372:71-9.

17. Scottish Intercollegiate Guidelines Network. Methodology checklist 2: randomised controlled trials. 2012. https://www. sign.ac.uk/media/1713/checklist_for_controlled_trials.doc. Cited 9 Nov 2020.

18. Hopkins W, Marshall S, Batterham A, Hanin J. Progressive statistics for studies in sports medicine and exercise science. Med Sci Sports Exerc. 2009;41(1):3-12.

19. Higgins JP, Thompson SG, Deeks JJ, Altman DG. Measuring inconsistency in meta-analyses. Br Med $\mathrm{J}$. 2003;327(7414):557-60.

20. Afsharnezhad T, Faghihi S, Hazrati A, Bahrami K. The effects of cold water immersion on anaerobic power, dynamic balance 
and muscle activation after a karate kumite fighting in female karateka. Int J Appl Exerc Physiol. 2017;6(3):72-9.

21. Chow G, Chung J, Fong S. Differential effects of post-exercise ice water immersion and room temperature water immersion on muscular performance, vertical jump, and agility in amateur rugby players: a randomized controlled trial. Sci Sports. 2018;33(6):e271-9.

22. Cook CJ, Beaven CM. Individual perception of recovery is related to subsequent sprint performance. Br J Sports Med. 2013;47(11):705-9.

23. Crowe M, O'Connor D, Rudd D. Cold water recovery reduces anaerobic performance. Int J Sports Med. 2007;28(12):994-8.

24. Crowther FA, Sealey RM, Crowe MJ, Edwards AM, Halson SL. Effects of various recovery strategies on repeated bouts of simulated intermittent activity. J Strength Cond Res. 2019;33(7):1781-94.

25. de Araújo L, da Silva D, Peserico C, Machado F. Effects of LED therapy and cryotherapy recovery methods on maximal isometric handgrip strength and blood lactate removal in Brazilian Jiu-Jitsu (BJJ) practitioners. Sci Sports. 2017;32(6):376-80.

26. Dunne A, Crampton D, Egaña M. Effect of post-exercise hydrotherapy water temperature on subsequent exhaustive running performance in normothermic conditions. J Sci Med Sport. 2013; 16(5):466-71.

27. Egaña M, Jordan L, Moriarty T. A 2.5 min cold water immersion improves prolonged intermittent sprint performance. J Sci Med Sport. 2019;22(12):1349-54.

28. Garcia C, Da Mota G, Marocolo M. Cold water immersion is acutely detrimental but increases performance post- $12 \mathrm{~h}$ in rugby players. Int J Sports Med. 2016;37(08):619-24.

29. Hamlin M. The effect of recovery modality on blood lactate removal and subsequent repetitive sprint performance in netball players. N Z J Sports Med. 2007;34(2):12-7.

30. Heyman E, De Geus B, Mertens I, Meeusen R. Effects of four recovery methods on repeated maximal rock climbing performance. Med Sci Sports Exerc. 2009;41(6):1303-10.

31. King M, Duffield R. The effects of recovery interventions on consecutive days of intermittent sprint exercise. J Strength Cond Res. 2009;23(6): 1795-802.

32. McCarthy A, Mulligan J, Egaña M. Postexercise cold-water immersion improves intermittent high-intensity exercise performance in normothermia. Appl Physiol Nutr Metab. 2016;41(11):1163-70.

33. Nunes RFH, Duffield R, Nakamura FY, Bezerra EDS, Sakugawa RL, Loturco I, et al. Recovery following Rugby Union matches: effects of cold water immersion on markers of fatigue and damage. Appl Physiol Nutr Metab. 2019;44(5):546-56.

34. Parouty J, Al Haddad H, Quod M, Leprêtre PM, Ahmaidi S, Buchheit M. Effect of cold water immersion on 100-m sprint performance in well-trained swimmers. Eur J Appl Physiol. 2010;109(3):483-90.

35. Pinho Jnr E, Brito CJ, Santos WC, Valido CN, Mendes EL, Franchini E. Influence of cryotherapy on muscle damage markers in jiu-jitsu fighters after competition: a cross-over study. Rev Andal Med Deport. 2014;7(1):7-12.

36. Santos WOC, Brito CJ, Pinho EAJ, Valio CN, Mendes EL, Nunes MAP, et al. Cryotherapy post-training reduces muscle damage markers in jiu-jitsu fighters. J Hum Sport Exerc. 2012;7(3):629-38.

37. Stearns RL, Nolan JK, Huggins RA, Maresh CM, Munõz CX, Pagnotta KD, et al. Influence of cold-water immersion on recovery of elite triathletes following the ironman world championship. J Sci Med Sport. 2018;21(8):846-51

38. Qu C, Wu Z, Xu M, Qin F, Dong Y, Wang Z, et al. Cryotherapy models and timing-sequence recovery of exercise-induced muscle damage in middle-and long-distance runners. J Athl Train. 2020;55(4):329-35.

39. Adamczyk JG, Krasowska I, Boguszewski D, Reaburn P. The use of thermal imaging to assess the effectiveness of ice massage and cold-water immersion as methods for supporting post-exercise recovery. J Therm Biol. 2016;60:20-5.

40. Amir N, Hashim H, Saha S. The effect of single bout of 15 minutes of 15-degree celsius cold water immersion on delayedonset muscle soreness indicators. Int Conf Mov Health Exerc. 2016;2016:45-51.

41. Argus CK, Broatch JR, Petersen AC, Polman R, Bishop DJ, Halson S. Cold-water immersion and contrast water therapy: no improvement of short-term recovery after resistance training. Int J Sports Physiol Perform. 2017;12(7):886-92.

42. Doeringer JR, Colas M, Peacock C, Gatens DR. The effects of postexercise cooling on muscle performance and soreness perception. Int J Athl Ther Train. 2018;23(2):73-6.

43. Eston R, Peters D. Effects of cold water immersion on the symptoms of exercise-induced muscle damage. J Sports Sci. 1999;17(3):231-8.

44. Goodall S, Howatson G. The effects of multiple cold water immersions on indices of muscle damage. J Sports Sci Med. 2008;7(2):235-41.

45. Hassan E. Thermal therapy and delayed onset muscle soreness. J Sports Med Phys Fit. 2011;51(2):249-54.

46. Hohenauer E, Costello JT, Deliens T, Clarys P, Stoop R, Clijsen R. Partial-body cryotherapy $\left(-135^{\circ} \mathrm{C}\right)$ and cold-water immersion $\left(10^{\circ} \mathrm{C}\right)$ after muscle damage in females. Scand $\mathrm{J}$ Med Sci Sports. 2019;30(3):485-95.

47. Howatson G, Goodall S, Van Someren K. The influence of cold water immersions on adaptation following a single bout of damaging exercise. Eur J Appl Physiol. 2009;105(4):615-21.

48. Jajtner AR, Hoffman JR, Gonzalez AM, Worts PR, Fragala MS, Stout JR. Comparison of the effects of electrical stimulation and cold-water immersion on muscle soreness after resistance exercise. J Sport Rehabil. 2015;24(2):99-108.

49. Jakeman J, Macrae R, Eston R. A single 10-min bout of coldwater immersion therapy after strenuous plyometric exercise has no beneficial effect on recovery from the symptoms of exercise-induced muscle damage. Ergonomics. 2009;52(4):456-60.

50. Machado A, Almeida A, Micheletti J, Vanderlei F, Tribst M, Netto Junior J, et al. Dosages of cold-water immersion post exercise on functional and clinical responses: a randomized controlled trial. Scand J Med Sci Sports. 2017;27(11):1356-63.

51. Missau E, Teixeira ADO, Franco OS, Martins CN, Paulitsch FDS, Peres W, et al. Cold water immersion and inflammatory response after resistance exercises. Rev Bras Med Esporte. 2018;24(5):372-6.

52. Paddon-Jones D, Quigley B. Effect of cryotherapy on muscle soreness and strength following eccentric exercise. Int J Sports Med. 1997;18(8):588-90.

53. Rose CL, Caillaud C, Edwards KM, Siegler J, Graham K. Does whole body cryotherapy improve muscle recovery after damaging eccentric exercise? J Aust Strength Cond. 2014;22(5):48-51.

54. Sánchez-Ureña B, Rojas-Valverde D, Gutiérrez-Vargas R. Effectiveness of two cold water immersion protocols on neuromuscular function recovery: a tensiomyography study. Front Physiol. 2018;9(766):1-7.

55. Siqueira AF, Vieira A, Bottaro M, Ferreira-Júnior JB, de Toledo NO, de Souza VC, et al. Multiple cold-water immersions attenuate muscle damage but not alter systemic inflammation and muscle function recovery: a parallel randomized controlled trial. Sci Rep. 2018;8(1):1-12. 
56. Vaile J, Halson S, Gill N, Dawson B. Effect of hydrotherapy on the signs and symptoms of delayed onset muscle soreness. Eur J Appl Physiol. 2008;102(4):447-55.

57. Vanderlei FM, Machado A, Netto JJ, Pastre C. Post-exercise recovery of biological, clinical and metabolic variables after different temperatures and durations of cold water immersion: a randomized clinical trial. J Sports Med Phys Fit. 2017;57(10):1267-75.

58. Vieira A, Siqueira AF, Ferreira-Júnior JB, Do Carmo J, Durigan JL, Blazevich A, et al. The effect of water temperature during cold-water immersion on recovery from exercise-induced muscle damage. Int J Sports Med. 2016;37(12):937-43.

59. Anderson D, Nunn J, Tyler CJ. Effect of cold $\left(14{ }^{\circ} \mathrm{C}\right)$ vs. ice $\left(5{ }^{\circ} \mathrm{C}\right)$ water immersion on recovery from intermittent running exercise. J Strength Cond Res. 2018;32(3):764-71.

60. Bailey D, Erith S, Griffin P, Dowson A, Brewer D, Gant N, et al. Influence of cold-water immersion on indices of muscle damage following prolonged intermittent shuttle running. J Sports Sci. 2007;25(11):1163-70.

61. Barber S, John P, Brown F, Hill J. The efficacy of repeated cold water immersion on recovery following a simulated rugby union protocol. J Strength Cond Res. 2020;34(12):3523-9.

62. Bosak A, Bishop P, Green J, Hawver G. Impact of cold water immersion on $5 \mathrm{~km}$ racing performance. Sport J. 2009;12(2):1-8.

63. Brophy-Williams N, Landers G, Wallman K. Effect of immediate and delayed cold water immersion after a high intensity exercise session on subsequent run performance. J Sports Sci Med. 2011;10(4):665-70.

64. Crowther F, Sealey R, Crowe M, Edwards A, Halson S. Influence of recovery strategies upon performance and perceptions following fatiguing exercise: a randomized controlled trial. BMC Sports Sci Med Rehabil. 2017;9(1):25-33.

65. Crystal NJ, Townson DH, Cook SB, LaRoche DP. Effect of cryotherapy on muscle recovery and inflammation following a bout of damaging exercise. Eur J Appl Physiol. 2013;113(10):2577-86.

66. Dantas G, Barros A, Silva B, Belém L, Ferreira V, Fonseca A, et al. Cold-water immersion does not accelerate performance recovery after $10-\mathrm{km}$ street run: randomized controlled clinical trial. Res Q Exerc Sport. 2020;91(2):228-38.

67. Delextrat A, Calleja-González J, Hippocrate A, Clarke ND. Effects of sports massage and intermittent cold-water immersion on recovery from matches by basketball players. J Sports Sci. 2013;31(1):11-9.

68. Elias GP, Varley MC, Wyckelsma VL, McKenna MJ, Minahan CL, Aughey RJ. Effects of water immersion on posttraining recovery in Australian footballers. Int J Sports Physiol Perform. 2012;7(4):357-66.

69. Elias GP, Wyckelsma VL, Varley MC, McKenna MJ, Aughey RJ. Effectiveness of water immersion on postmatch recovery in elite professional footballers. Int J Sports Physiol Perform. 2013;8(3):243-53.

70. Fonseca LB, Brito CJ, Silva RJS, Silva-Grigoletto ME, da Silva WM, Franchini E. Use of cold-water immersion to reduce muscle damage and delayed-onset muscle soreness and preserve muscle power in jiu-jitsu athletes. J Athl Train. 2016;51(7):540-9.

71. Getto CN, Golden G. Comparison of active recovery in water and cold-water immersion after exhaustive exercise. Athl Train Sports Health Care. 2013;5(4):169-76.

72. Higgins TR, Cameron ML, Climstein M. Acute response to hydrotherapy after a simulated game of rugby. J Strength Cond Res. 2013;27(10):2851-60.

73. Higgins TR, Climstein M, Cameron M. Evaluation of hydrotherapy, using passive tests and power tests, for recovery across a cyclic week of competitive rugby union. J Strength Cond Res. 2013;27(4):954-65.
74. Ingram J, Dawson B, Goodman C, Wallman K, Beilby J. Effect of water immersion methods on post-exercise recovery from simulated team sport exercise. J Sci Med Sport. 2009;12(3):417-21.

75. Jones B, Lander J, Brubaker D. The effects of different recovery interventions following a repeated rugby union (sevens) game simulated protocol. J Aust Strength Cond. 2013;21(4):5-13.

76. Lane $\mathrm{KN}$, Wenger $\mathrm{H}$. Effect of selected recovery conditions on performance of repeated bouts of intermittent cycling separated by 24 hours. J Strength Cond Res. 2004;18(4):855-60.

77. Leeder JD, Van Someren KA, Bell PG, Spence JR, Jewell AP, Gaze D, et al. Effects of seated and standing cold water immersion on recovery from repeated sprinting. J Sports Sci. 2015;33(15):1544-52.

78. Leeder JD, Godfrey M, Gibbon D, Gaze D, Davison GW, Van Someren KA, et al. Cold water immersion improves recovery of sprint speed following a simulated tournament. Eur J Sport Sci. 2019;19(9):1166-74.

79. Lindsay A, Carr S, Cross S, Petersen C, Lewis JG, Gieseg SP. The physiological response to cold-water immersion following a mixed martial arts training session. Appl Physiol Nutr Metab. 2017;42(5):529-36.

80. Minett GM, Duffield R, Billaut F, Cannon J, Portus MR, Marino FE. Cold-water immersion decreases cerebral oxygenation but improves recovery after intermittent-sprint exercise in the heat. Scand J Med Sci Sports. 2014;24(4):656-66.

81. Moreira A, Costa EC, Coutts AJ, Nakamura FY, da Silva DA, Aoki MS. Cold water immersion did not accelerate recovery after a futsal match. Rev Bras Med Esporte. 2015;21(1):40-3.

82. Pointon M, Duffield R. Cold water immersion recovery after simulated collision sport exercise. Med Sci Sports Exerc. 2012;44(2):206-16.

83. Pournot H, Bieuzen F, Duffield R, Lepretre P-M, Cozzolino C, Hausswirth C. Short term effects of various water immersions on recovery from exhaustive intermittent exercise. Eur J Appl Physiol. 2011;111(7):1287-95.

84. Rupp AK, Selkow MN, Parente RW, Ingersoll DC, Weltman LA, Saliba AS. The Effect of Cold Water Immersion on 48-Hour Performance Testing in Collegiate Soccer Players. J Strength Cond Res. 2012;26(8):2043-50.

85. Stenson MC, Stenson MR, Matthews TD, Paolone VJ. 5000 meter run performance is not enhanced $24 \mathrm{hrs}$ after an intense exercise bout and cold water immersion. J Sports Sci Med. 2017;16(2):272-9.

86. Tabben M, Ihsan M, Ghoul N, Coquart J, Chaouachi A, Chaabene $\mathrm{H}$, et al. Cold water immersion enhanced athletes' wellness and 10-m short sprint performance $24-\mathrm{h}$ after a simulated mixed martial arts combat. Front Physiol. 2018;9(1542):1-8.

87. Takeda M, Sato T, Hasegawa T, Shintaku H, Kato H, Yamaguchi $\mathrm{Y}$, et al. The effects of cold water immersion after rugby training on muscle power and biochemical markers. J Sports Sci Med. 2014;13(3):616-23.

88. Vaile J, Halson S, Gill N, Dawson B. Effect of hydrotherapy on recovery from fatigue. Int J Sports Med. 2008;29(7):539-44.

89. White GE, Rhind SG, Wells GD. The effect of various cold-water immersion protocols on exercise-induced inflammatory response and functional recovery from high-intensity sprint exercise. Eur J Appl Physiol. 2014;114(11):2353-67.

90. Wiewelhove T, Schneider C, Döweling A, Hanakam F, Rasche C, Meyer T, et al. Effects of different recovery strategies following a half-marathon on fatigue markers in recreational runners. PLoS ONE. 2018;13(11):1-18.

91. Ertel KA, Hallam JE, Hillman AR. The effects of training status and exercise intensity on exercise-induced muscle damage. J Sports Med Phys Fit. 2020;60(3):449-55.

92. Wilson GJ, Murphy AJ. The use of isometric tests of muscular function in athletic assessment. Sports Med. 1996;22(1):19-37. 
93. Wilson GJ, Murphy AJ, Pryor JF. Musculotendinous stiffness: its relationship to eccentric, isometric, and concentric performance. J Appl Physiol. 1994;76(6):2714-9.

94. Halder A, Gao C. Muscle cooling and performance: a review. Eur J Sports Med. 2015;2(1):39-48.

95. Ihsan M, Watson G, Abbiss CR. What are the physiological mechanisms for post-exercise cold water immersion in the recovery from prolonged endurance and intermittent exercise? Sports Med. 2016;46(8):1095-109.

96. Stephens JM, Halson S, Miller J, Slater GJ, Askew CD. Coldwater immersion for athletic recovery: one size does not fit all. Int J Sports Physiol Perform. 2017;12(1):2-9.

97. Vogelaere P, Deklunder G, Lecroart J. Cardiac output variations in supine resting subjects during head-out cold water immersion. Int J Biometeorol. 1995;39(1):40-5.

98. Wiewelhove T, Fernandez-Fernandez J, Raeder C, Kappenstein J, Meyer T, Kellmann M, et al. Acute responses and muscle damage in different high-intensity interval running protocols. J Sports Med Phys Fit. 2016;56(5):606-15.

99. Ihsan M, Watson G, Lipski M, Abbiss CR. Influence of postexercise cooling on muscle oxygenation and blood volume changes. Med Sci Sports Exerc. 2013;45(5):876-82.

100. Cheung K, Hume PA, Maxwell L. Delayed onset muscle soreness. Sports Med. 2003;33(2):145-64.

101. Kellmann M. Preventing overtraining in athletes in high-intensity sports and stress/recovery monitoring. Scand J Med Sci Sports. 2010;20:95-102.

102. Trojian TH, Beedie CJ. Placebo effect and athletes. Curr Sports Med Rep. 2008;7(4):214-7.

103. Nosaka K, Newton M, Sacco P. Delayed-onset muscle soreness does not reflect the magnitude of eccentric exercise-induced muscle damage. Scand J Med Sci Sports. 2002;12(6):337-46.

104. Beedie CJ. Placebo effects in competitive sport: qualitative data. J Sports Sci Med. 2007;6(1):21-8.

105. Viitasalo J, Niemelä K, Kaappola R, Korjus T, Levola M, Mononen $\mathrm{H}$, et al. Warm underwater water-jet massage improves recovery from intense physical exercise. Eur J Appl Physiol Occup Physiol. 1995;71(5):431-8.
106. Greenham G, Buckley JD, Garrett J, Eston R, Norton K. Biomarkers of physiological responses to periods of intensified, non-resistance-based exercise training in well-trained male athletes: a systematic review and meta-analysis. Sports Med. 2018;48(11):2517-48.

107. Thompson HS, Scordilis SP, De Souza MJ. Serum creatine kinase activity varies with ovulatory status in regularly exercising, premenopausal women. Horm Res Paediatr. 2006;65(3):151-8.

108. Magal M, Dumke CL, Urbiztondo ZG, Cavill MJ, Triplett NT, Quindry JC, et al. Relationship between serum creatine kinase activity following exercise-induced muscle damage and muscle fibre composition. J Sports Sci. 2010;28(3):257-66.

109. Baird MF, Graham SM, Baker JS, Bickerstaff GF. Creatine kinase and exercise-related muscle damage implications for muscle performance and recovery. J Nutr Metab. 2012;2012:1-13.

110. Callegari GA, Novaes JS, Neto GR, Dias I, Garrido ND, Dani C. Creatine kinase and lactate dehydrogenase responses after different resistance and aerobic exercise protocols. J Hum Kinet. 2017;58:65-72.

111. Rodrigues BM, Dantas E, de Salles BF, Miranda H, Koch AJ, Willardson JM, et al. Creatine kinase and lactate dehydrogenase responses after upper-body resistance exercise with different rest intervals. J Strength Cond Res. 2010;24(6):1657-62.

112. Chen TC, Lin K-Y, Chen H-L, Lin M-J, Nosaka K. Comparison in eccentric exercise-induced muscle damage among four limb muscles. Eur J Appl Physiol. 2011;111(2):211-23.

113. Jamurtas AZ, Theocharis V, Tofas T, Tsiokanos A, Yfanti C, Paschalis V, et al. Comparison between leg and arm eccentric exercises of the same relative intensity on indices of muscle damage. Eur J Appl Physiol. 2005;95(2):179-85.

114. Siqueira LDO, Muccini T, Dall Agnol I, Filla L, Tibbola P, Luvison A, et al. Serum chemistry test and urinalysis parameter analysis in half marathon athletes. Arq Bras Endocrinol Metabol. 2009;53(7):844-52.

\section{Authors and Affiliations}

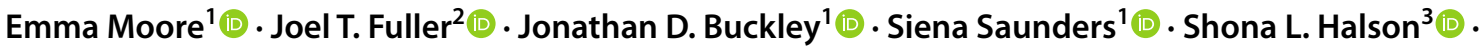 James R. Broatch ${ }^{4}$ (i) . Clint R. Bellenger ${ }^{1}$ (i)}

Emma Moore

emma.moore@mymail.unisa.edu.au

1 Alliance for Research in Exercise, Nutrition and Activity (ARENA), University of South Australia, Adelaide, SA, Australia

2 Faculty of Medicine, Health and Human Sciences, Macquarie University, Sydney, NSW, Australia
3 School of Behavioural and Health Sciences, McAuley at Banyo, Brisbane, QLD, Australia

4 Institute for Health and Sport (IHES), Victoria University, Footscray, VIC, Australia 